OPEN ACCESS

Edited by:

Carina Almeida,

Instituto Nacional Investigaciao Agraria e Veterinaria (INIAV), Portugal

Reviewed by:

Sarah Maddocks,

Cardiff Metropolitan University,

United Kingdom

Laura Cerqueira,

University of Porto, Portugal

Pilar Teixeira,

University of Minho, Portugal

*Correspondence:

Makrina Totsika

makrina.totsika@qut.edu.au

Specialty section:

This article was submitted to

Biofilms,

a section of the journal

Frontiers in Cellular and

Infection Microbiology

Received: 07 January 2022

Accepted: 04 February 2022

Published: 25 February 2022

Citation:

Hawas S, Verderosa $A D$ and Totsika M (2022) Combination Therapies for Biofilm Inhibition and Eradication: A Comparative Review of Laboratory and Preclinical Studies. Front. Cell. Infect. Microbiol. 12:850030. doi: 10.3389/fcimb.2022.850030

\section{Combination Therapies for Biofilm Inhibition and Eradication: A Comparative Review of Laboratory and Preclinical Studies}

\author{
Sophia Hawas ${ }^{1,2}$, Anthony D. Verderosa ${ }^{1,2}$ and Makrina Totsika ${ }^{1,2 *}$ \\ ${ }^{1}$ Centre for Immunology and Infection Control, Queensland University of Technology, Brisbane, QLD, Australia, ${ }^{2}$ School of \\ Biomedical Sciences, Queensland University of Technology, Brisbane, QLD, Australia
}

Microbial biofilms are becoming increasingly difficult to treat in the medical setting due to their intrinsic resistance to antibiotics. To combat this, several biofilm dispersal agents are currently being developed as treatments for biofilm infections. Combining biofilm dispersal agents with antibiotics is emerging as a promising strategy to simultaneously disperse and eradicate biofilms or, in some cases, even inhibit biofilm formation. Here we review studies that have investigated the anti-biofilm activity of some well-studied biofilm dispersal agents (e.g., quorum sensing inhibitors, nitric oxide/nitroxides, antimicrobial peptides/amino acids) in combination with antibiotics from various classes. This review aims to directly compare the efficacy of different combination strategies against microbial biofilms and highlight synergistic treatments that warrant further investigation. By comparing across studies that use different measures of efficacy, we can conclude that treating biofilms in vitro and, in some limited cases in vivo, with a combination of an anti-biofilm agent and an antibiotic, appears overall more effective than treating with either compound alone. The review identifies the most promising combination therapies currently under development as biofilm inhibition and eradication therapies.

Keywords: antimicrobial resistance (AMR), anti-biofilm, infection, nitroxides, antibiotics, nitric oxide (NO), quorum sensing inhibitors (QSI), antimicrobial peptides (AMPs)

\section{INTRODUCTION}

When planktonic bacterial cells contact a surface, whether biotic or abiotic, they can irreversibly attach to it, proliferate, and form complex three-dimensional communities known as biofilms (Vestby et al., 2020). Biofilms are inherently tolerant to environmental stress, antimicrobials, and host immune responses (McDougald et al., 2011; Vestby et al., 2020). Consequently, biofilms pose a major clinical challenge as biofilm-related infections are extremely difficult to treat or permanently eradicate, with very few viable treatments or management options available (Azevedo et al., 2020; Vishwakarma, 2020). Current strategies for managing biofilm-related infections are aimed at: (i) preventing biofilm formation, (ii) limiting biofilm expansion, or (iii) biofilm eradication by chemical or mechanical means (e.g. removal) (Vuotto and Donelli, 2019; Zhao et al., 2019 Azevedo et al., 2020). 
Achieving complete biofilm eradication with antibiotics alone is extremely challenging, with even clinically significant bacterial reduction being often hard to achieve. The precise mechanism by which conventional antibiotics fail to eradicate biofilms is not fully understood and has been the topic of extensive investigation (Gilbert et al., 2002; Lewis, 2008; Høiby et al., 2010). Restricted drug penetration resulting from the presence of a protective extracellular matrix, reduced cell growth, and the presence of persister cells within biofilms (quiescent cells exhibiting extreme antimicrobial tolerance) are all thought to contribute to the high antibiotic tolerance of biofilms (McDougald et al., 2011). To address this challenge, several new and innovative strategies have come under intense investigation (Barraud et al., 2015; Brackman and Coenye, 2015; Pletzer and Hancock, 2016; Fleming and Rumbaugh, 2017). Many of these, such as the development of biofilm inhibition, dispersal, and eradication agents, have been extensively reviewed elsewhere (Fleming and Rumbaugh, 2017; Roy et al., 2018; Verderosa et al., 2019c; Ghosh et al., 2020). Here we focus on studies whereby biofilm dispersal agents are coadministered with antimicrobials and evaluated as combination treatment strategies against bacterial biofilms.

Biofilm dispersal agents rarely possess inherent antimicrobial activity. As such, their potential use as biofilm treatment strategies requires supplementation with an effective antimicrobial agent to successfully disperse and eradicate biofilms (Fleming and Rumbaugh, 2018). Several promising classes of biofilm dispersal agents have been reported to date (Kaplan, 2010); however, the true therapeutic potential of dispersal agents lies in their ability to restore or synergistically enhance the activity of commonly prescribed antimicrobials. In clinical settings, co-treatment is imperative as biofilm dispersal alone would result in translocation of live bacteria to new sites in the body and the subsequent seeding of new infection foci (Fleming and Rumbaugh, 2018). Upon combining a dispersal agent with an effective antibiotic/antimicrobial, the combined treatment both disperses and eradicates biofilm-residing cells, thus preventing further dissemination (Figure 1).

This review aims to collate and compare studies that have evaluated biofilm dispersal and eradication combination treatments against established biofilms from clinically relevant pathogens. Important details of the studies cited in this review have been summarised in a comprehensive table (Table S1), where the efficacy of the standalone treatments (biofilm dispersal agent or antibiotic) was compared to the efficacy of the combination treatment as reported within each study. For studies where the combination efficacy was greater than the sum of the standalone strategies, these have been included in a summary table (Table 1) showcasing the most promising antibiofilm combination treatments currently under intense study.

\section{CELL-SIGNALLING DISRUPTERS}

\section{Quorum Sensing Inhibitors (QSIs)}

Quorum sensing (QS) is a bacterial communication system which allows neighbouring cells to send and receive signal molecules, called autoinducers, in a density-dependent manner. QS has been shown to play a pivotal role in biofilm regulation for many species (Brackman and Coenye, 2015). Targeting QS with inhibitors (QSIs) has been a significant innovation in the antibiofilm field, even though the role of QSIs in biofilm formation and dispersal is not always fully understood for different Gram-positive and Gram-negative bacteria (Brackman
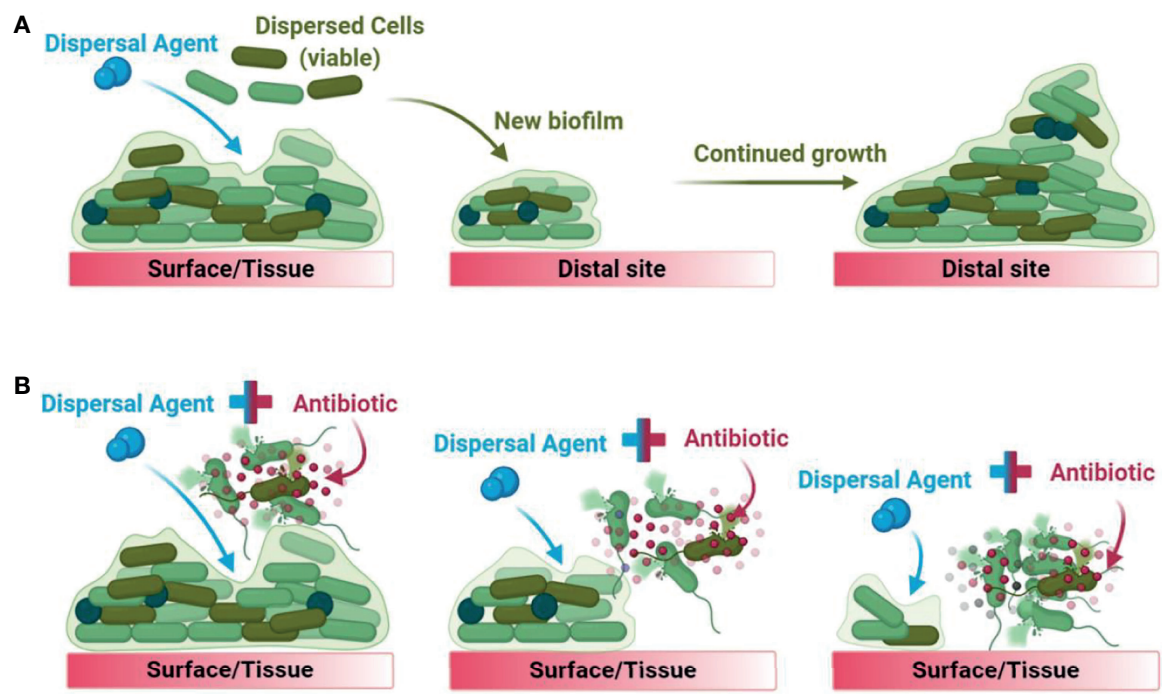

Continuous combination treatment

FIGURE 1 | Graphical summary of the downstream consequences of treating a biofilm with dispersal agents alone (A) versus with a biofilm dispersal-eradication combination strategy (B), demonstrating how it is clinically and industrially relevant. 
TABLE 1 | List of promising combination treatments against bacterial biofilms currently in development.

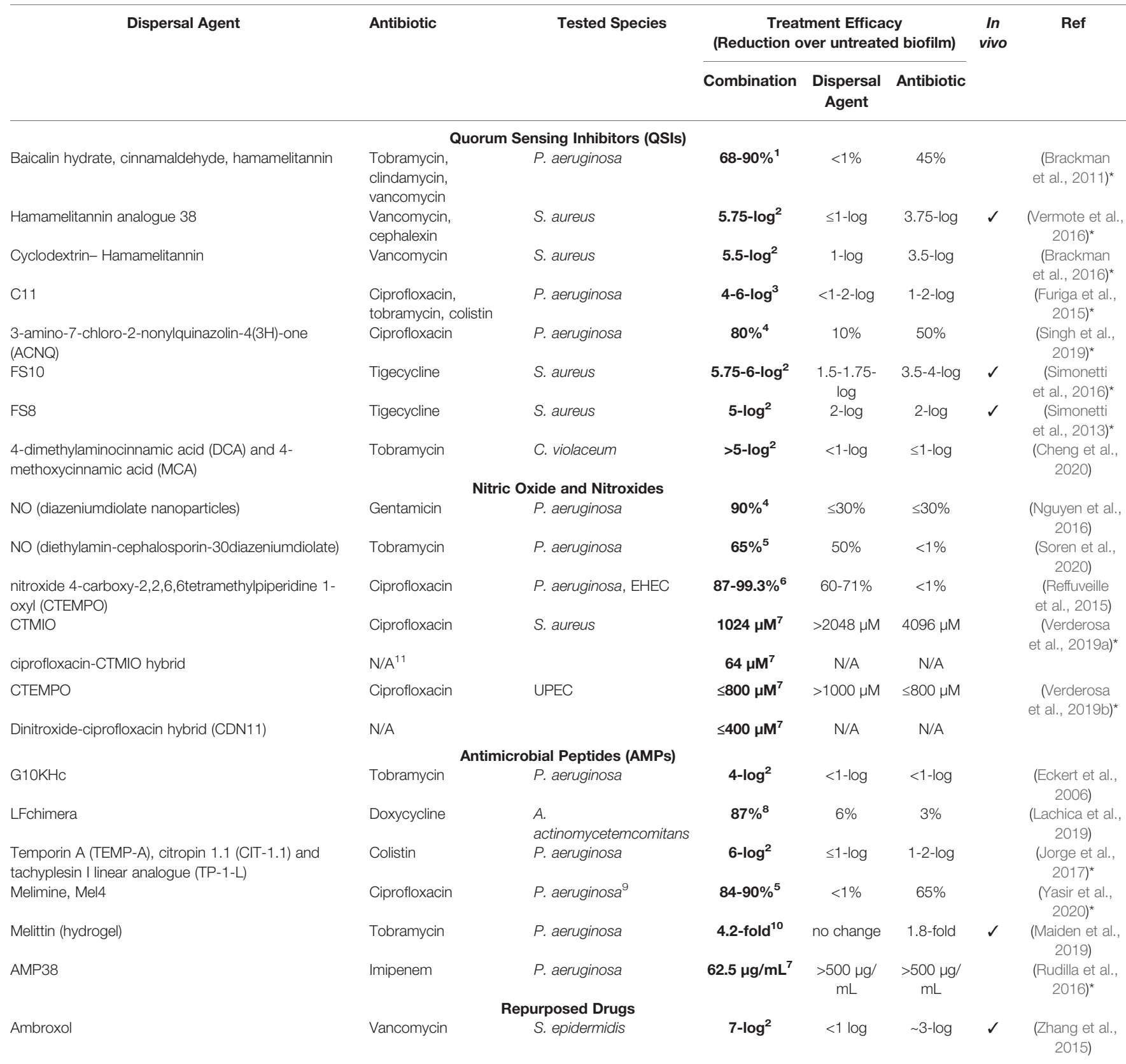

${ }^{1} \%$ reduction in total biofilm colony forming units (CFU).

${ }^{2}$ log reduction in CFU/mL.

${ }^{3}$ log reduction in biofilm CFU/cm².

${ }^{4} \%$ reduction in viable biofilm bacteria (CFU).

$5 \%$ reduction in total biofilm biomass.

${ }^{6} \%$ Biofilm eradication (CFU).

${ }^{7}$ Minimum Biofilm Eradication Concentration (MBEC).

${ }^{8} \%$ reduction biofilm $\mathrm{CFU} / \mathrm{cm}^{2}$.

${ }^{9}$ against ciprofloxacin-sensitive isolates.

${ }^{10}$ fold reduction in biofilm bioluminescence.

${ }^{11}$ N/A denotes hybrid compound testing, hybrids already contained the antibiotic and were not tested in combination with additional antibiotics.

*Low cytotoxicity to human cell lines or in tested in vivo model.

All studies investigating biofilm co-treatments cited in this review were compiled into a table comparing the efficacy of the dispersal agent, antibiotic alone, and combination treatment (Table S1). Where co-treatment was more effective than the sum of the standalone treatments, the combination treatment was deemed promising, and these studies are summarized here under different dispersal agent groups (QSIs, NO/Nitroxides, AMPS, Repurposed Drugs). Other study details provided include the name of the dispersal agent(s) and antibiotic(s), bacterial species tested, and if the combination treatment was tested against biofilm infection in vivo. Efficacy measures for each study are different (as marked in the combination column and relevant footnote); efficacy measures reported are in relation to untreated biofilm controls in all studies. 
and Coenye, 2015). QS inhibition can occur by inhibiting autoinducer synthesis, degradation of signalling molecules, interfering with signal binding, and inhibition of the signal transduction cascade, which results in dysregulated biofilm signalling and subsequently, dispersal or inhibition of the biofilm (Brackman and Coenye, 2015; Jiang et al., 2019). Most QSIs are derived from proteins (autoinducers, transcription factors and regulators) that mediate QS in the bacterial target, acting as competitive inhibitors of these systems.

Several studies have been conducted using QSIs in combination with antibiotics to either inhibit and/or eradicate biofilms. One of the earliest studies, conducted by Brackman et al., focused on the efficacies of baicalin hydrate, cinnamaldehyde, and hamamelitannin (structures shown in Figure 2) in combination with the antibiotics tobramycin, clindamycin, and vancomycin (Brackman et al., 2011). These compounds were tested against established (24-hour) Pseudomonas aeruginosa and Staphylococcus aureus biofilms in Mueller-Hinton agar (Brackman et al., 2011). Initial in vitro testing showed that individual treatment with inhibitor or antibiotic alone was largely ineffective $(<10 \%$ reduction in biofilm bacterial numbers) against $P$. aeruginosa ATCC 9027 and S. aureus CS1 and Mu50. Tobramycin alone reduced only $P$. aeruginosa $\mathrm{PAO} 1$ biofilm bacterial numbers by $45 \%$ (Brackman et al., 2011). When biofilms were treated with a combination of QSI and antibiotic, viable bacteria for all strains showed a $68-90 \%$ reduction (Table 1), except S. aureus Mu50, which were only reduced by $6 \%$ when treated with clindamycin and a QSI (Brackman et al., 2011). This strain has been shown to be resistant to clindamycin planktonically, which may explain reduced efficacy also against its biofilms (Cui et al., 2009).

These QSI-antibiotic combinations were also evaluated in vivo against Burkholderia cenocepacia and Burkholderia multivorans, in a Caenorhabditis elegans and Galleria mellonella survival model and in a mouse lung infection model (Brackman et al., 2011). QSIs alone exacted a strain-dependent effect on the survival of $G$. mellonella, with a minimal protective effect observed against the Burkholderia spp. (0-30\% survival) but very high protection in larvae infected with $S$. aureus (70$100 \%$ survival) (Brackman et al., 2011). In C. elegans, none of the QSIs alone exhibited any protective effect ( $<10 \%$ survival) (Brackman et al., 2011). Standalone antibiotic treatment in $C$. elegans showed increased survival compared to the inhibitor only, and this was also observed for G. mellonella (Brackman et al., 2011). Combination treatment exhibited the most protective effect, with most combinations resulting in $80-100 \%$ survival when infected with either $P$. aeruginosa and $S$. aureus. These outcomes were further confirmed in the mouse model, which showed that the combination of tobramycin $(30 \mathrm{mg} / \mathrm{kg})$ and baicalin hydrate $(2 \mathrm{mg} / \mathrm{kg})$ reduced pulmonary bacterial numbers by $99.9 \%$ (Brackman et al., 2011).

The same authors extended their investigation of hamamelitannin, by testing it in combination with several antibiotics (vancomycin, cefazolin, cefalonium, cephalexin, cefoxitin, daptomycin, linezolid, tobramycin, fusidic acid) against established S. aureus biofilms (Brackman et al., 2016). Here they measured the efficacy of single treatment (antibiotic only) and compared it to combination treatment (antibiotic and hamamelitannin). For all antibiotics, combination treatment was at least equally as effective but, in most cases, resulted in greatly increased efficacy $(40-70 \%$ additional biofilm eradication compared to antibiotic treatment alone) (Brackman et al., 2016). The most effective combinations were hamamelitannin with cefazolin, cefoxitin, tobramycin or fusidic acid, which resulted in $\geq 90 \%$ eradication of the biofilm (Brackman et al., 2016). Similarly, Vermote et al. derived a hamamelitannin analogue (compound-38) that was reported to have a 20 -fold lower median minimum bactericidal $\left(\mathrm{MBC}_{50}\right)$ value against $S$. aureus Mu50 planktonic cells (Vermote et al., 2016). Mirroring the initial hamamelitannin study (Brackman et al., 2011), all combinations were at least as, or more effective than their standalone counterpart (Vermote et al., 2016). The most effective combinations were compound-38 with vancomycin, cefazolin, daptomycin or tobramycin ( $\geq 90 \%$ eradication, Table 1) (Vermote et al., 2016). Combined, these studies<smiles>O=C(O)[C@H]1O[C@@H](Oc2cc3oc(-c4ccccc4)cc(=O)c3c(O)c2O)[C@H](O)[C@@H](O)[C@H]1O</smiles>

1

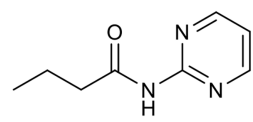

4<smiles>O=C/C=C/c1ccccc1</smiles>

2

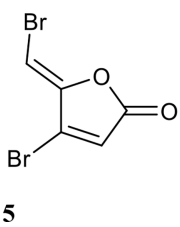

FIGURE 2 | Chemical structures of QSIs 1 baicalin, 2 cinnamaldehyde, 3 hamamelitannin, 4 N- (2-pyrimidyl)butanamide (C11), and 5 furanone C-30. 
suggest that hamamelitannin is most effective against biofilm bacteria when administered with the antibiotics cefazolin and tobramycin (Brackman et al., 2016; Vermote et al., 2016).

In a different study, Brackman et al. incorporated hamamelitannin into a delivery system to improve its efficacy against S. aureus biofilms (Brackman et al., 2016). They achieved this by incorporating the inhibitor into a cyclodextrin complex which could release the QSI and an antibiotic at a controlled rate (Brackman et al., 2016). Utilising these complexes as a delivery system increased efficacy in vitro, and the use of the delivery system with hamamelitannin alone reduced biofilm CFU by 1 log (Brackman et al., 2016). However, when the system was used in combination with both vancomycin and hamamelitannin, biofilm CFUs were reduced by 5.5 logs (Table 1) (Brackman et al., 2016). Hamamelitannin thus appears to demonstrate a broad-spectrum of biofilm-eradication potentiating activity when administered as a co-treatment with antibiotics. Furthermore, it synergised well with antibiotics from different classes. These properties make hamamelitannin one of the more promising QSIs reviewed here, warranting further exploration into its synergistic capabilities. Given that hamamelitannin has already been tested in vivo in mice, this warrants further investigation into its potential toxicity and side effects, before moving into clinical development. As successful analogues with similar efficacies have also been developed (Vermote et al., 2016), this dispersal agent has potential to be modified chemically as well.

Other promising QSIs have also been tested in combination with antibiotics against biofilms. Some of these include furanone C-30 and C11 (structures shown in Figure 2), ajoene (garlic extract), 3-amino-7-chloro-2-nonylquinazolin-4(3H)-one (ACNQ) (PqsR receptor inhibitor), derivatives of cinnamic acid, horseradish extract and alkylquinolone-based inhibitors. Christensen et al. investigated combinations of tobramycin and the inhibitors furanone C-30, ajoene, and horseradish extract against $P$. aeruginosa biofilm infection in vivo using a mouse intraperitoneal implant model (Christensen et al., 2012). Tobramycin alone failed to reduce bacterial numbers by more than $1 \log$, but combination treatment reduced bacteria by up to 3 logs (Christensen et al., 2012). Treatment with inhibitor alone however, also showed similar efficacy, suggesting that the interaction between the two compounds was additive rather than synergistic. A derivative of a signalling molecule in $P$. aeruginosa QS, C11, was also tested in combination with the antibiotics ciprofloxacin, tobramycin, ceftazidime, and colistin against forming P. aeruginosa biofilms (Furiga et al., 2015). Treatment with individual agents reduced biofilm surface area $\left(\mathrm{CFU} / \mathrm{cm}^{2}\right)$ by $1-2$ logs, while combination treatment with antibiotics and C11 resulted in a 4-6-log reduction (Table 1) (Furiga et al., 2015). The only combination which was not synergistic was ceftazidime and $\mathrm{C} 11$, reducing biofilm surface area only by 1-2 logs (Furiga et al., 2015).

Building upon this work, Singh et al. used an inhibitor similar to C11 which also targets QS in Pseudomonas spp (Singh et al., 2019). Using engineered polymeric nanoparticles as a delivery system, they co-delivered the inhibitor ACNQ $(4 \mu \mathrm{g} / \mathrm{mL})$ and ciprofloxacin $(60 \mu \mathrm{g} / \mathrm{mL})$ to treat established $P$. aeruginosa biofilms (Singh et al., 2019). Individual treatments with the inhibitor and antibiotic were somewhat effective (10\% and 50\% eradication, respectively), however, combination treatment significantly enhanced eradication of established biofilms $(80 \%$ eradication; Table 1) (Singh et al., 2019). Using an alternative delivery system, Ho et al. tested the alkylquinolone QSI [1] (20 $\mu \mathrm{M})$ in combination with tobramycin $(25 \mu \mathrm{g} / \mathrm{mL})$ to treat $P$. aeruginosa biofilms (24-hours) (Ho et al., 2020). Here they employed a squalenyl hydrogen sulfate nanoparticle delivery system to deliver both compounds at the same time (Ho et al., 2020). Treatment with the antibiotic alone resulted in a 4-5-log reduction in biofilm CFU compared to the untreated control, regardless of the mode of delivery (Ho et al., 2020). Combination treatment with the delivery system achieved complete eradication ( $>6-\log$ reduction), whereas combination treatment without the delivery system resulted in 3-4-log less eradication (Ho et al., 2020). Thus, the design of the delivery system is important for combination treatment with QSIs. The dual nature of a combination treatment suggests that for it to be successful, both compounds must be in the same environment at the same time to work effectively, as depicted in Figure 1B. As standalone treatments do not need to interact with any other compounds, this may be why the delivery system had no impact on their efficacy, as reported in this study. This should be taken into consideration when designing future co-treatment strategies.

Another studied QSI is baicalin (Figure 2), a flavonoid isolated from the roots of Scutellaria baicalensis (Chinese skullcap) (Slachmuylders et al., 2018). Using this compound, Slachmuylders et al. examined biofilm eradication in combination with tobramycin, gentamicin, kanamycin or neomycin (Slachmuylders et al., 2018). Tobramycin was the most effective against several strains of Burkholderia spp. when combined with baicalin hydrate $(75-95 \%$ increase in biofilm eradication against 5 out of 9 strains tested, compared to antibiotic treatment alone) (Slachmuylders et al., 2018). Similarly, Luo et al. tested baicalin in combination with levofloxacin, amikacin, and ceftazidime against $P$. aeruginosa biofilms (Luo et al., 2017). Amikacin and baicalin were the most effective combination for biofilm inhibition in vitro, while in vivo (mouse peritoneal implant model), ceftazidime and baicalin were the most effective combination for reducing bacterial numbers (Luo et al., 2017).

Another series of QS inhibitors shown to be active against S. aureus are analogues of the RNA-III inhibiting peptide (RIP) (Cirioni et al., 2013; Simonetti et al., 2013; Simonetti et al., 2016). Mouse studies using the QSIs FS3 and FS8 with daptomycin and tigecycline, respectively, demonstrated additive and synergistic efficacy against S. aureus biofilms (Cirioni et al., 2013; Simonetti et al., 2013). In these studies, Cirioni et al. and Simonetti et al. implanted grafts (with or without inhibitor) and injected $S$. aureus into the graft site while the antibiotic was administered intraperitoneally (Cirioni et al., 2013; Simonetti et al., 2013; Simonetti et al., 2016). Treatment with the QSI or antibiotic alone resulted in a 2-3-log reduction in bacterial numbers after 7 days of infection, compared to the untreated control group (Cirioni et al., 2013; Simonetti et al., 2013). 
Treatment with both compounds resulted in an additive effect for FS3 and daptomycin (4-log reduction) and a synergistic effect for FS8 and tigecycline (5-log reduction, Table 1) (Cirioni et al., 2013; Simonetti et al., 2013). Following on from this, FS10 was also tested in combination with tigecycline and showed similar results in the same mouse infection model against both methicillin-susceptible S. aureus (MSSA) and MRSA (Simonetti et al., 2016). Groups treated with FS10 alone showed minimal CFU reduction after 7 days post implantation (1-2-log reduction), while groups treated with tigecycline only showed higher log reduction in bacterial numbers (3.5-4 logs, Table 1) (Simonetti et al., 2016). Combination treatment was the most efficacious against both MSSA and MRSA, reducing bacterial numbers by 5.5-6 logs (Simonetti et al., 2016). Considering that the inhibitor and antibiotic are administered separately, administration of both at the same site could potentially improve efficacy.

Lastly, cinnamic acid, a metabolite of Cinnamomum cassia (Chinese cinnamon), has recently been shown to inhibit quorum sensing in bacteria (Vasconcelos et al., 2018). In a study by Cheng et al., two synthesised cinnamic acid derivatives, 4dimethylaminocinnamic acid (DCA) and 4-methoxycinnamic acid (MCA) were tested in combination with tobramycin against Chromobacterium violaceum biofilms (Cheng et al., 2020). Individual treatments were largely ineffective at eradicating established biofilms ( $\leq 1-\log$ reduction in biofilm CFU counts), though combination treatment was highly successful and resulted in a 5-log CFU reduction compared to the untreated control, see Table 1 (Cheng et al., 2020). It is also noteworthy that the core structures of these compounds are amenable to synthetic modification, which makes them ideal candidates for the development of more potent derivatives. In conjunction with this, additional in vivo/ex vivo studies should be conducted to test the toxicity of newly modified inhibitors while also assessing discrepancies between in vitro vs. in vivo effects.

Overall, quorum sensing inhibitors are a promising class of biofilm disruptors. Most QSI studies to date demonstrate that while they are not effective at eradicating biofilms on their own, when used together with antibiotics they can effectively eradicate established biofilms both in vitro and in vivo. Continued research into QSIs is sorely needed, as many of the mechanisms inhibited by them are poorly understood (Brackman and Coenye, 2015). Additionally, further manipulation of the structures of these inhibitors would open many avenues of therapeutic development, considering their amenability to synthetic modification.

\section{Nitric Oxide}

Nitric oxide (NO) is a free radical colourless gas (at room temperature), and a well-established signalling molecule in eukaryotic organisms (Barraud et al., 2015). One of the earliest uses of $\mathrm{NO}$ as a biofilm dispersal agent was documented by Barraud et al. (2006) and since then its potential as a biofilm dispersal agent has been widely documented. While NO concentrations at the $\mathrm{mM}$ range are antibacterial (Barraud et al., 2015), inhalation of NO gas at such high concentrations is also acutely toxic to the respiratory tract of humans (Weinberger et al., 2001). However, NO-mediated biofilm dispersal occurs at concentrations sublethal for bacteria (nM range) (Barraud et al., 2006). Thus, the use of $\mathrm{NO}$ as an effective biofilm eradication strategy requires supplementation with an antimicrobial agent. NO signals dispersal of bacterial biofilms by interacting with enzymes that affect intracellular concentrations of bis- $\left(3^{\prime}-5^{\prime}\right)$-cyclic dimeric guanosine monophosphate (c-diGMP) (Williams and Boon, 2019). The molecular mechanism was recently characterised in some species of bacteria, including $P$. aeruginosa, Nitrosomonas europaea, and Shewanella oneidensis (Hossain and Boon, 2017; Hossain et al., 2017; Nisbett et al., 2019). Interaction of NO with NO-sensitive enzymes stimulates the activity of phosphodiesterases (PDEs), which degrade c-di-GMP and signal biofilm dispersal (McDougald et al., 2011).

Several studies have tested NO for its ability to potentiate antibiotics in vitro (Barraud et al., 2009; Nguyen et al., 2016; Liu et al., 2020; Soren et al., 2020). NO is a difficult molecule to work with due to its gaseous form at room temperature and high reactivity (Barraud et al., 2015). Hence, most studies have used NO donors, such as sodium nitroprusside (SNP) and (Z)-1-[NMethyl-N-[6-(N-methylammoniohexyl)amino]]diazen-1-ium1,2-diolate (MAHMA NONOate) (Barraud et al., 2009; Barnes et al., 2013; Marvasi et al., 2015) (structure shown in Figure 3), which albeit are also known to be inherently unstable molecules (Wang et al., 2002). In order to address this, several NO delivery

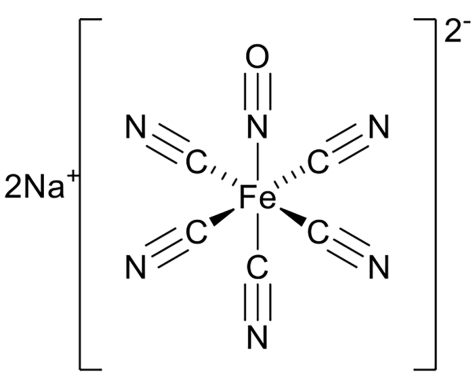

6

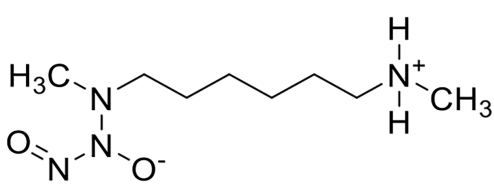

7

FIGURE 3 | Chemical structures of 6 sodium nitroprusside (SNP) and 7 (Z)-1-[N-Methyl-N-[6-(N-methylammoniohexyl)amino]diazen-1-ium-1,2-diolate (MAHMA NONOate), 
systems have been reported to release NO in a controlled manner at the target site (Lu et al., 2013; Nguyen et al., 2016; Ren et al., 2016; Liu et al., 2020). Examples of these include diazeniumdiolate (NONOate), micro/nanoparticles, and amphiphilic poly(amidoamine) (PAMAM) dendrimers (Lu et al., 2013; Nguyen et al., 2016; Soren et al., 2020). These systems improve handling and dose mediation, prevent or limit off-target effects, and increase the efficacy of biofilm dispersal by NO while minimising cytotoxicity to the host (Davies et al., 2001; Findlay et al., 2004; Poh et al., 2017; Yang et al., 2018; Verderosa et al., 2019c). In this section, we review studies that have combined NO with antibiotics for either dispersing or eradicating clinically relevant biofilms, while excluding studies which have only tested $\mathrm{NO}$ alone in vitro.

In one of the earlier in vitro studies, Barraud et al. investigated the potentiation of tetracycline by $\mathrm{NO}$ against biofilms from a variety of different bacterial species, using the NO-donor sodium nitroprusside (SNP) (Barraud et al., 2009). Pre-treating Vibrio cholerae biofilms with $\mathrm{NO}$ and then adding tetracycline reduced the biofilm surface area by $90 \%$ compared to untreated biofilms (Barraud et al., 2009). NO alone reduced the biofilm surface area by $67 \%$, while tetracycline alone only afforded a $21 \%$ biofilm reduction (Barraud et al., 2009). The study also investigated the ability of NO to potentiate chlorine used for water treatment. Similar to tetracycline, NO treatment of $V$. cholerae biofilms followed by chlorine resulted in significant biofilm reduction (85-90\%) compared to untreated controls (Barraud et al., 2009). The authors concluded that NO could enhance the activity of tetracycline and chlorine against biofilms when administered sequentially (NO treatment followed by tetracycline/chlorine treatment), however co-treatment with $\mathrm{NO}$ and tetracycline was not investigated, nor was the viability of the dispersed cells.

While sequential treatment of biofilms with a dispersal agent followed by an antibiotic is informative and may find use in industrial settings, such as water treatment, this approach would not be suited to the clinical setting. Dispersing a clinical biofilm prior to antimicrobial intervention can lead to systemic bacterial dissemination, which can result in spread of infection, septicaemia, and bacteraemia (Fleming and Rumbaugh, 2018; Müsken et al., 2018). For this reason, subsequent biofilm studies have focused on NO co-treatment with antibiotics.

NO co-administration with antibiotics has been facilitated by delivery systems such as those developed by Soren et al. which use the prodrug diethylamin-cephalosporin- $\underline{3} 0$ diazeniumdiolate (DEA-C3D) to investigate the delivery of $\mathrm{NO}$ in combination with tobramycin to eradicate $P$. aeruginosa biofilms (Barraud et al., 2012; Soren et al., 2020). DEA-C3D belongs to a class of NO-donor prodrugs known as cephalosporin-30-diazeniumdiolates (C3Ds), which contain the phenacetyl side chain of the first-generation cephalosporin cefaloram and the NO donor diazeniumdiolate (Soren et al., 2020). These compounds deliver NO by local release upon contact with bacterial $\beta$-lactamase enzymes (Soren et al., 2020). When used to deliver NO for the treatment of $P$. aeruginosa biofilms, NO alone reduced biofilm biomass by $50 \%$, while treatment with the antibiotic tobramycin alone had no effect on biofilm biomass. However, $\mathrm{NO}$ and tobramycin co-delivery reduced biofilm biomass by $65 \%$. While the potentiation of tobramycin was relatively modest, it should be noted that the concentration of antibiotic used in the study was sublethal (Soren et al., 2020), suggesting that efficacy could be likely increased by using tobramycin at higher concentrations. A similar potentiation trend was also observed for colistin. Despite colistin treatment alone being extremely effective against $P$. aeruginosa biofilms $(90 \%$ biomass reduction), co-treatment with DEA-C3D resulted in almost complete biofilm eradication (98\% reduction, Table 1) (Soren et al., 2020). The design of this NO delivery strategy is certainly innovative; however, its application is limited to $\beta$-lactamase-producing bacteria, and furthermore, it is unfortunate that the antibacterial properties of the delivery system $(\beta$-lactam) are lost upon NO release, which was an intentional design feature (Soren et al., 2020). The authors comment that the ultimate goal of this delivery system is to deliver NO to a targeted site, containing $\beta$-lactamase-producing bacteria, to reduce tissue damage from NO's reactivity (Soren et al., 2020). In addition, as the study determined biofilm eradication by visualising remaining cells, dispersed cells were not studied. This makes it difficult to evaluate the efficacy of this treatment in the context of a "disperse and eradicate" strategy, as it is important to determine whether dispersed cells remained viable or were killed.

Another study targeting $P$. aeruginosa biofilms used a diazeniumdiolate nanoparticle delivery system in combination with the antibiotic gentamicin (Nguyen et al., 2016). Here, Nguyen and co-workers designed and produced a polymeric nanoparticle delivery system that simultaneously released NO and gentamicin at a target site by placing a gentamicinNONOate complex (generated by reacting gentamicin and NO gas) at the core of the nanoparticle (Nguyen et al., 2016). Cotreatment with $\mathrm{NO}$ and gentamicin reduced biofilm viability by $90 \%$, whereas treatment with the individual compounds only reduced viability by $\leq 30 \%$, (Table 1) (Nguyen et al., 2016). These results are promising and should the compound prove to be nontoxic to mammalian cells, a property that was not examined, the strategy would certainly merit in vivo analysis. Similarly, Liu et al. engineered a NO delivery system using a derivative of a naturally occurring compound, chitosan (Liu et al., 2020). The donor, chitosan-graft-poly(amidoamine) dendrimer (CS-PAMAM), was used to deliver methicillin and NO to methicillin-resistant Staphylococcus aureus (MRSA) biofilms (Liu et al., 2020). They observed that despite the strains being methicillin resistant, cotreatment with methicillin increased biofilm eradication a further $10 \%$ than with NO treatment alone (Liu et al., 2020). This study is unique as the target pathogen has acquired resistance to the antibiotic yet co-treatment with $\mathrm{NO}$ had an effect, which we speculate suggests that NO was likely dispersing cells from the biofilm outer layer and allowing higher levels of methicillin to access and kill underlying biofilm-residing cells.

The above studies have showcased the promising potential of $\mathrm{NO}$ as a biofilm dispersal agent with and without antibiotics in vitro. However, studies on NO co-treatments of clinical biofilms using in vivo models (cystic fibrosis, urinary tract infections, chronic wounds, etc. (Vestby et al., 2020)] are currently lacking. Nevertheless several in vivo studies on $\mathrm{NO}$ have provide valuable 
insight into its potential use as an infection treatment and or control strategy (Webert et al., 2000; Miller et al., 2012; Miller et al., 2013).

Inhaled NO has been studied as a standalone treatment for pneumonia in rats and has more recently progressed to phase I human trials (Webert et al., 2000; Miller et al., 2012; Miller et al., 2013; Deppisch et al., 2016). A rat model of P. aeruginosa pneumonia was initially used to test the efficacy of inhaled NO (Webert et al., 2000; Miller et al., 2012; Miller et al., 2013). Rats were injected intratracheally with $10^{8}$ colony forming units (CFU) of $P$. aeruginosa and then monitored over time for any physiological changes and for bacterial carriage in the lungs (Webert et al., 2000). The study successfully demonstrated that NO could reduce the pulmonary bacterial load after 24 hours of NO inhalation at a concentration of $40 \mathrm{ppm}$ (Webert et al., 2000). A follow up study by the same group demonstrated that an even higher concentration of $\mathrm{NO}$ (160 ppm) given intermittently was also effective at lowering viable $P$. aeruginosa counts in the lungs of infected rats (Miller et al., 2013). This confirmed previous in vitro studies, which had shown that at a concentration of $160 \mathrm{ppm}$, NO is bactericidal (Schairer et al., 2012). The success of these preclinical animal studies paved the way for a small Phase I study on 10 healthy adult volunteers, which confirmed the same dosing regimen of 160 ppm for 30 minutes was safe (Miller et al., 2012). A follow-up pilot clinical study treated eight cystic fibrosis patients suffering from long term (>6 months) bacterial and/or fungal lung infection(s) with gaseous NO for 30 minutes, three times daily, at a concentration of $160 \mathrm{ppm}$ for two periods of five days over two weeks (Deppisch et al., 2016). Patients were followed over the course of seven months and post-NO treatment were all found to have an average log-reduction of 3.6 and 3.0, respectively, of bacteria and fungi (regardless of species) in their sputum samples (Deppisch et al., 2016). In addition to this, ESBL-producing Escherichia coli or Aspergillus spp. biofilms were undetectable after treatment. This study is one of the first of its kind and is unique from other NO dispersal studies in that it did not require the use of a NO delivery system. Additionally, these studies demonstrate the safety of NO administration even at bactericidal concentrations, which are higher than the concentrations shown to be required to disperse bacterial biofilms in vitro. Future clinical studies will greatly enhance the development of new respiratory therapeutics with potential benefits for other respiratory diseases.

\section{Nitroxides}

The development of antibacterial and antibiofilm therapies based on NO is often limited by its high reactivity, instability, and inherent human toxicity at high concentrations (Barraud et al., 2015; Verderosa et al., 2019c). Thus, alternatives that mimic the behaviour of NO, but lack its limitations, have recently been explored (de la Fuente-Núñez et al., 2013; O'Loughlin et al., 2013; Rajasekaran et al., 2019).

Nitroxides are structurally similar to $\mathrm{NO}$ as both contain an unpaired electron delocalised over the nitrogen-oxygen bond. However, unlike NO, nitroxides are generally air-stable crystalline solids at room temperature, which makes their handling and delivery far more convenient than NO. Furthermore, nitroxides have been recently shown to exhibit similar antibiofilm properties to NO in vitro (de la Fuente-Núñez et al., 2013; Verderosa et al., 2016). The antibiofilm properties of nitroxides were first explored by de la Fuente Núñez et al., who showed that nitroxides not only mimicked the biofilm dispersal activity of $\mathrm{NO}$ but also prevented biofilm formation in vitro (de la Fuente-Núñez et al., 2013). In a follow up study by the same group, nitroxides were shown to potentiate the action of ciprofloxacin against both $P$. aeruginosa and E. coli biofilms (Reffuveille et al., 2015). Here, they investigated the ability of the nitroxide 4-carboxy-2,2,6,6tetramethylpiperidine 1-oxyl (CTEMPO) (structure shown in Figure 4) to potentiate the activity of ciprofloxacin when administered as a co-treatment (Reffuveille et al., 2015). CTEMPO $(20 \mu \mathrm{M})$ alone dispersed $P$. aeruginosa and enterohemorrhagic E. coli (EHEC) biofilms resulting in significantly reduced biomass $(60 \%$ and $71 \%$, respectively, Table 1) (Reffuveille et al., 2015). At the same concentration, CTEMPO also potentiated ciprofloxacin activity against $P$. aeruginosa and EHEC biofilms, eradicating $99.3 \%$ and 93\%, respectively (Reffuveille et al., 2015). This occurred at a ciprofloxacin concentration of just $320 \mathrm{ng} / \mathrm{mL}$ for $P$. aeruginosa and $20 \mathrm{ng} / \mathrm{mL}$ for EHEC when ciprofloxacin treatment alone had

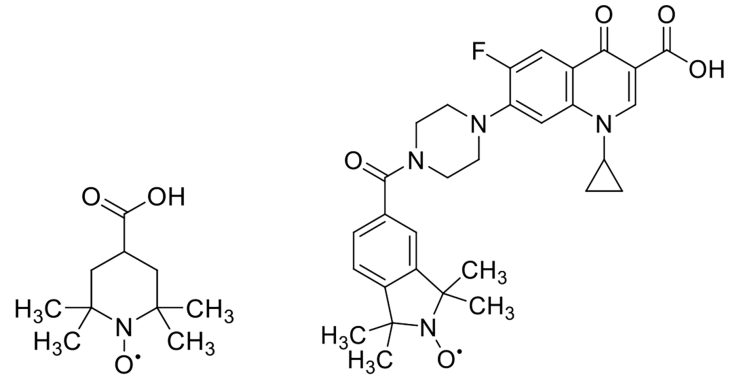

8
9

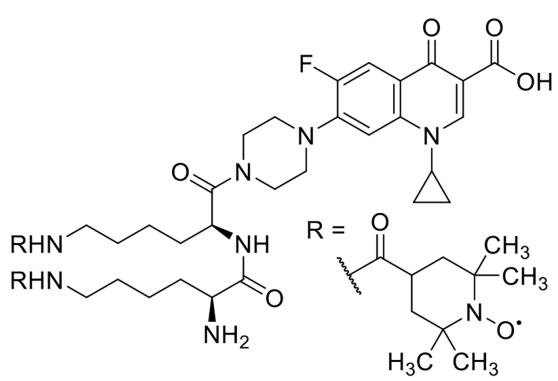

10

FIGURE 4 | Chemical structures of 8 4-carboxy-2,2,6,6tetramethylpiperidine 1-oxyl (CTEMPO), 9 ciprofloxacin-nitroxide hybrid-27 and 10 dinitroxide-ciprofloxacin hybrid $11(\mathrm{CDN}-11)$ 
no or minimal effect on biofilm biovolume (Reffuveille et al., 2015). Dispersed cells from CTEMPO treated $P$. aeruginosa biofilms were also collected and quantified at 3-, 6- and 24hours post treatment. Between the 3 and 6 hour treatment timepoints, there was minimal change in bacterial numbers, however after 24 hours, there was a minimum 5 -fold change in dispersed bacterial counts (Reffuveille et al., 2015). This suggests that after 24 hours of CTEMPO treatment, the biofilm has been sufficiently dispersed that many cells are becoming dislodged and flowing out of the system.

Verderosa et al. built upon this work by synthetically linking the nitroxide to the ciprofloxacin moiety to create a variety of ciprofloxacin-nitroxide hybrids (Verderosa et al., 2016; Verderosa et al., 2017; Verderosa et al., 2019a; Verderosa et al., 2019b). These hybrid compounds were effective in dispersing and eradicating both Gram-negative ( $P$. aeruginosa, uropathogenic E. coli (UPEC)) as well as Gram-positive (S. aureus) biofilms in vitro (Verderosa et al., 2017; Verderosa et al., 2019a; Verderosa et al., 2019b). P. aeruginosa biofilms were $94 \%$ eradicated with ciprofloxacin-nitroxide hybrid-27 (structure shown in Figure 4) treatment at $20 \mu \mathrm{M}$ (Verderosa et al., 2017). Treatment with the hybrid was on par with the synergy reported previously against $P$. aeruginosa biofilms using CTEMPO and ciprofloxacin cotreatment (Reffuveille et al., 2015). However, hybrid compounds provide some intrinsic advantages over co-treatments, such as $i$ ) more favourable pharmacokinetic and pharmacodynamic properties and $i$ i) insurance that the dual action of dispersal and eradication are contained in the one compound, which is especially important for in vivo studies (Verderosa et al., 2017; Fleming and Rumbaugh, 2018). In a follow-up study by Verderosa et al., co-treatment and treatment with hybrid compounds was tested against $S$. aureus biofilms (Verderosa et al., 2019a). Cotreatment with nitroxide ( $8 \mu \mathrm{M}$ for CTEMPO) and ciprofloxacin $(256 \mu \mathrm{M})$ was effective at eradicating biofilms, affording a 16 -fold improvement (Table 1) in the minimum biofilm eradication concentration (MBEC) (99.9\% eradication) compared to ciprofloxacin treatment alone (Verderosa et al., 2019a). Interestingly, treatment with the ciprofloxacin-nitroxide hybrid27 at $64 \mu \mathrm{M}$ was 4 -fold more potent than co-treatment and 64 -fold more potent than ciprofloxacin alone, suggesting that hybrid compounds (dispersal agent linked to antibiotic) may provide distinct advantages over co-treatments against some species (Verderosa et al., 2019a). In a subsequent study, the authors developed a synthetic strategy for altering the ratio of nitroxide to antibiotic producing a dinitroxide-ciprofloxacin hybrid (CDN11, structure shown in Figure 4) with potent activity against UPEC biofilms in vitro (Verderosa et al., 2019b). CDN-11 was effective in eradicating biofilm residing UPEC, with a $99 \%$ reduction in bacterial numbers (vs. untreated group) at $12.5 \mu \mathrm{M}$ (Table 1) (Verderosa et al., 2019b). The use of nitroxides in the eradication of biofilms is certainly encouraging, especially considering how well they can potentiate the activity of ciprofloxacin (either as a co-treatment or in hybrid compounds). However, their ability to potentiate the activity of other classes of antibiotics remains to be explored as does their antibiofilm activity in vivo. The lack of human cell toxicity reported for nitroxides and nitroxide functionalised antibiotics (Sadowska-Bartosz et al., 2015; Verderosa et al., 2017) however supports their further development as antibiofilm antimicrobials.

Clearly, NO and nitroxides are effective biofilm dispersal agents and based on in vitro and in vivo studies, they both hold genuine promise as treatment strategies for biofilm related infections. As co-treatment agents they have also showed synergy with several antibiotics and proved to be effective at eradicating established biofilms. While the combined use of NO, nitroxides and antibiotics is still in early preclinical testing, its development towards clinical applications is likely to progress. Continued assessment against other biofilm-forming organisms (in vitro and in vivo) is needed to ascertain how broad-spectrum these combinations can be. NO has already been tested against several well established biofilm-forming species, including the fungal pathogen Candida albicans, and proven efficacious for biofilm dispersal (Barraud et al., 2009). Further work expanding the testing of such combination treatments towards several other species would aid both their development as therapeutics and understanding of the mechanism behind NO's biofilm dispersing ability, which is likely to differ between microbes.

\section{PEPTIDES/MEMBRANE DISRUPTERS}

\section{Antimicrobial Peptides}

Antimicrobial peptides (AMPs) are small cationic and amphipathic molecules of 12-50 amino acids that have the ability to disrupt bacterial membranes (Grassi et al., 2017). AMPs exhibit a broad-spectrum of activity and have a high potential to target metabolically dormant cells in biofilms (Grassi et al., 2017). AMPs are often studied in combination with antibiotics and other antibiofilm compounds, and many of these studies have been briefly described by Grassi et al. in 2017 (Grassi et al., 2017). Here, we report the efficacy afforded through combination treatment compared to standalone and also include studies that have been published since then.

An early study on AMP-antibiotic combinations assessed the synergistic activity of the synthetic AMP G10KHc (amino acid sequence shown in Table 2) in combination with tobramycin for the eradication of $P$. aeruginosa biofilms (Eckert et al., 2006). Treatment with $\mathrm{G} 10 \mathrm{KHc}$ or tobramycin alone (each at $100 \mu \mathrm{g} /$ $\mathrm{mL}$ ) for 4 and 24 hours had little effect on established biofilms (Eckert et al., 2006). However, when used together at the same concentrations, a 4-log reduction in bacterial numbers (Table 1) was observed after 4 hours of co-treatment, with no viable CFU recovered from the biofilms after 24 hours (Eckert et al., 2006). The same group further investigated how G10KHc potentiated tobramycin activity by staining cells with propidium iodide (PI) and observing whether the dye was present in treated cells, as it cannot enter cells with intact membranes (Krishan, 1975; Eckert et al., 2006). Bacterial cells treated with G10KHc were found to fluoresce, indicating that the peptide disrupted the cell membrane, subsequently allowing tobramycin to enter and kill cells (Eckert et al., 2006). 
TABLE 2 | Amino acid sequences of antimicrobial peptides (AMPs) with notable antibiofilm activity.

\begin{tabular}{|c|c|c|}
\hline Peptide Name & Type & Sequence \\
\hline G10KHc & Synthetic & KKHRKHRKHRKHGGSGGSKNLRRIIRKGIHIIKKYG \\
\hline Nisin-A & Natural & $\begin{array}{l}\text { MSTKDFNLDLVSVSKKDSGASPRITSIS } \\
\text { LCTPGCKTGALMGCNMKTATCHCSIHVSK }\end{array}$ \\
\hline Melittin & Natural & $\mathrm{NH}_{2}$-GIGAVLKVLTTGLPALISWIKRKRQQ-CONH ${ }_{2}$ \\
\hline 1018 & Synthetic & VRLIVAVRIWRR-NH $\mathrm{N}_{2}$ \\
\hline DJK-6 & Synthetic & VQWRRIRWWVIR-CONH${ }_{2}$ \\
\hline
\end{tabular}

\section{Naturally Derived Antimicrobial Peptides}

A wide variety of inherently antimicrobial peptides exist in nature serving as innate defence proteins against bacteria and produced by many species. Tachyplesin III, for example, is a short peptide from Southeast Asian horseshoe crabs that is similar in structure to the protegrin peptide family, which is also known for its antimicrobial properties (Minardi et al., 2007). Minardi et al. investigated the synergy between Tachyplesin III and piperacillin-tazobactam to treat $P$. aeruginosa biofilms in a rat ureteral stent model (Minardi et al., 2007). Treatment with either Tachyplesin III (10 mg/L stent coating) or piperacillintazobactam $(120 \mathrm{mg} / \mathrm{kg}$ intraperitoneally) resulted in a 3-log reduction of bacterial numbers compared to untreated controls (Minardi et al., 2007). Administered together however, the combination treatment resulted in a 5-log reduction of bacterial numbers, demonstrating synergistic activity (Minardi et al., 2007). In a subsequent study, the AMP BMAP-28 was used in combination with vancomycin to treat Enterococcus faecalis and S. aureus biofilms (Orlando et al., 2008). The co-treatment was tested both in vitro and in vivo, again in the rat stent model (Orlando et al., 2008). In vitro, a 4-fold decrease in MBEC value was observed, compared to individual treatments (Orlando et al., 2008). In vivo, stent cultures taken 5 days post implantation and urine cultures taken 24 hours post implantation showed a 2-3 log reduction in bacterial numbers for both bacterial species when compared to individual treatment groups, and a 5-log reduction compared to untreated controls (Orlando et al., 2008). Despite the agreement between in vitro and in vivo results, it is important to note that they are not directly comparable, as the methods for quantifying biofilm eradication were different (MBEC vs CFU quantification).

Similar to Tachyplesin III, lactoferrin is another peptide with antibacterial and bacterial anti-adhesion properties, and is commonly found in human blood and secreted fluids (Ammons and Copié, 2013). Wakabayashi et al. showed that treatment of Porphyromonas gingivalis biofilms with a combination of lactoferrin and ciprofloxacin was very effective, reducing biofilm biomass by $80 \%(0.5 \mathrm{mg} / \mathrm{mL}$ lactoferrin, $10 \mu \mathrm{g} /$ $\mathrm{mL}$ ciprofloxacin) whereas the compounds alone only reduced biofilm biomass by $50 \%$ and $40 \%$, respectively (Wakabayashi et al., 2009). Following on from this work, Lachica et al. tested lactoferrin chimera (LFchimera) against Aggregatibacter actinomycetemcomitans biofilms, a major causative agent of periodontitis (Lachica et al., 2019). Treatment with LFchimera alone or in combination with doxycycline was assessed for biofilm surface area. Combination treatment was most effective, reducing surface area by $87 \%$ compared to the untreated control, while individual treatments only reduced area by $<10 \%$, (Table 1) (Lachica et al., 2019).

Another naturally derived AMP, nisin, has also been assessed for antibiotic potentiation against biofilms in vitro. Nisin is a 34 amino acid peptide that originates from Lactococcus lactis and is commonly used as a food preservative (amino acid sequence shown in Table 2) (Tong et al., 2014) as it has been shown to have strong antimicrobial activity against many Gram-positive bacteria (Tong et al., 2014). Tong et al. investigated the synergy between nisin and several antibiotics for the eradication of $E$. faecalis biofilms in vitro (Tong et al., 2014). All nisin-antibiotic combinations were more effective than individual treatments, except for sulphapyridine, metronidazol, and polymyxin, which demonstrated no improved activity over standalone treatment (Tong et al., 2014). Conversely, for Gram-negative bacteria, Field et al. found that combining nisin with polymyxin and colistin was effective in preventing $P$. aeruginosa biofilm formation (Field et al., 2016). Biofilm formation was only slightly inhibited by the compounds alone at sub-MIC levels, but the combination of nisin (at $1 / 4 \mathrm{MIC}$ ) and polymyxin or colistin (at either $1 / 2$ or $1 / 5$ MIC) completely prevented biofilm growth up to 24 hours (Field et al., 2016). The difference between these two studies suggests that nisin is more synergistic with colistin over polymyxin, despite the antibiotics belonging to the same class. Colistin is a membrane-disrupting antibiotic that serves as a last resort treatment for multidrug resistant infections (Bialvaei and Samadi Kafil, 2015). Synergy between AMPs and colistin was also investigated by Jorge et al, using the AMPs temporin A (TEMP-A), citropin 1.1 (CIT-1.1) and tachyplesin I linear analogue (TP-1-L). AMPs were tested individually and in combination with colistin against $P$. aeruginosa and $S$. aureus biofilms (Jorge et al., 2017). All AMP-antibiotic combinations were effective in inhibiting biofilm formation by at least $2 \log$ $\left(\mathrm{CFU} / \mathrm{cm}^{2}\right.$ ) (Table 1) (Jorge et al., 2017). The most effective combination was colistin with CIT-1.1, which inhibited formation of $P$. aeruginosa (PAO1) biofilms by 7.7 logs (Jorge et al., 2017). All combinations were also tested for eradication activity against 24-hour established biofilms with only colistin and TP-1-L reported to completely eradicate (6-log reduction in bacterial numbers) P. aeruginosa biofilms (Jorge et al., 2017). All other combinations and individual treatments only reduced bacterial numbers by 2 logs or less (Jorge et al., 2017), which suggests that these colistin-AMP combinations are more useful for inhibiting rather than eradicating biofilms. These findings were supported by Mataraci et al., who tested several AMPs (indolicidin, CAMA (cecropin (1-7)-melittin A (2-9) amide), and nisin with multiple antibiotics (daptomycin, linezolid, 
teicoplanin, ciprofloxacin, and azithromycin) to prevent the formation of methicillin-resistant $S$. aureus biofilms in vitro (Mataraci and Dosler, 2012). All combination treatments (AMP/AMP, AMP/antibiotic and antibiotic/antibiotic) were equally effective at preventing biofilm formation (Mataraci and Dosler, 2012). These findings suggest that biofilms are more susceptible to combination treatment during early development stages. This may be due to lack or reduced biofilm features known to contribute to intrinsic resistance [i.e. a matrix of extracellular polymeric substances (EPS) and persister cells (Verderosa et al., 2019c)], allowing access of AMPs and antibiotics to actively growing biofilm cells.

Kalsy et al. investigated antibiotic combinations with the insect derived peptide cecropin A, which is involved in innate immune defence (Kalsy et al., 2020). Cecropin A was tested for synergy with nalidixic acid against uropathogenic E. coli (UPEC) biofilms (Kalsy et al., 2020). Interestingly, cecropin A was highly effective at inhibiting both forming and established biofilms, but combination with nalidixic acid did not improve its eradication activity (Kalsy et al., 2020), supporting the tenet that AMPs in general appear to be more effective at inhibiting biofilm formation over eradication. The fractional inhibitory concentration (FIC) index for cecropin A and nalidixic acid was calculated using the FIC formula (Figure 5) (Hall et al., 1983), which was reported $<0.5$ (indicating synergy), which conflicts with their previous findings (Kalsy et al., 2020). The authors state that UPEC was unlikely to harbour intrinsic resistance to the combination treatment, as its mode of action disrupts the bacterial outer membrane and overcoming this would be genetically and metabolically taxing (Kalsy et al., 2020). They then tested cecropin A and nalidixic acid in vivo using a G. mellonella (greater wax moth) larvae infection model (Kalsy et al., 2020). Combination treatment of moth larvae was also not protective against UPEC challenge (Kalsy et al., 2020). However, when a protease inhibitor was co-administered, all larvae treated with both cecropin A $(50 \mu \mathrm{g} / \mathrm{mL})$ and nalidixic acid $(0.5 \mathrm{ng} / \mathrm{mL})$ survived up to six days post injection with $E$. coli (Kalsy et al., 2020). They proposed that the addition of the protease inhibitor prevented proteolytic degradation of cecropin A in vivo, allowing it to have maximal activity in combination with nalidixic acid (Kalsy et al., 2020).

Thappeta et al., investigated the efficacy of the naturally derived chitosan-based peptide CSM5-K5 (structure shown in Figure 6) against $S$. aureus biofilms in combination with the antibiotics oxacillin, meropenem or streptomycin (Thappeta et al., 2020). MBC values were 2-3 fold lower with co-treatment compared to standalone treatment, a finding that was confirmed in vivo against $S$. aureus, E. faecalis and uropathogenic E. coli in a mouse wound excision model (Thappeta et al., 2020). The authors also reported little resistance development after 15 days of serially passaging the bacteria in vitro in the presence of CSM5-K5 (Thappeta et al., 2020). Similarly, Yasir et al. reported no resistance development to the AMPs melimine and Mel4 against $P$. aeruginosa biofilms after 30 days of exposure at sub-MIC levels (Yasir et al., 2020). Melimine is a cationic chimera of two naturally occurring peptides: melittin and protamine, and Mel4 is a derivative of melimine with demonstrated antibiofilm activity against $P$. aeruginosa (Yasir et al., 2020). Synergy between these AMPs and the antibiotic ciprofloxacin was assessed by measuring reduction in biomass in established (24-hour) ciprofloxacin resistant or sensitive $P$. aeruginosa biofilms (Yasir et al., 2020). Treatment with each peptide or ciprofloxacin alone at $1 \mathrm{x}$ MIC had no effect on ciprofloxacin resistant biofilms, but combination treatment at $1 \mathrm{x}$ MIC resulted in 61-66\% reduction in biofilm mass (Table 1) (Yasir et al., 2020). Synergy was also reported against ciprofloxacin sensitive biofilms, where peptide only treatment at 1x MIC had no effect, and while ciprofloxacin reduced biofilm mass by $65 \%$, combination treatment reduced biofilm mass by $84-90 \%$ (Yasir et al., 2020).

Recent advances in clinical treatment include the use of hydrogels as scaffolds for long-term drug release in wounds, to maintain wound sterility and aid healing (Azevedo et al., 2020). Maiden et al. investigated the integration of the AMP melittin (amino acid sequence shown in Table 2) in an agarose-based hydrogel in addition to co-treatment with the antibiotic tobramycin. Using an in vivo mouse wound model, they reported that tobramycin alone reduced biofilm bioluminescence (used here as a measure of biomass) by 1.8-fold after 4 hours, with melittin having no effect (Maiden et al., 2019). However, incorporating both compounds into the hydrogels reduced biofilm bioluminescence 4.2-fold (Table 1) (Maiden et al., 2019). Other studies incorporating hydrogels as delivery systems have also reported increased combination treatment efficacy, potentially due to simultaneous release of compounds, and engineered slow release of the compounds over time (Marvasi et al., 2015; Anjum et al., 2018; Maiden et al., 2019).

\section{A $B$ \\ $\frac{\mathrm{A}}{\mathrm{MIC}_{\mathrm{A}}}+\frac{\mathrm{B}}{\mathrm{MIC}_{\mathrm{B}}}=\mathrm{FIC}_{\mathrm{A}}+\mathrm{FIC}_{\mathrm{B}}=$ FIC Index Value}

FIGURE 5 | Fractional inhibitory concentration (FIC) index formula. The equation used to calculate synergy, indifference, or antagonism between two compounds

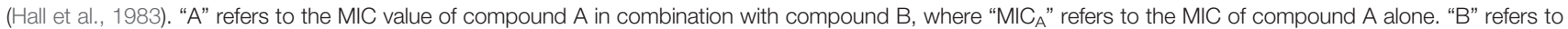
the MIC value of compound $B$ in combination with compound $A$, where "MIC ${ }_{B}$ " refers to the MIC of compound $B$ alone. These values added together output the FIC index value, where $<0.5$ indicates synergy, 0.5-4 indifference, and $>4$ antagonism. 


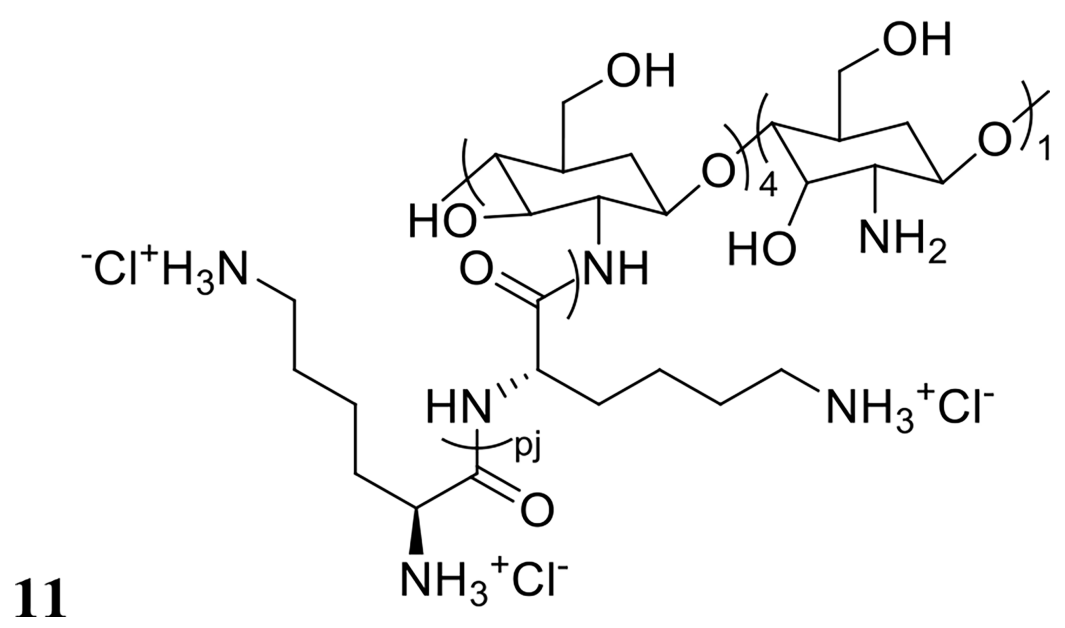

FIGURE 6 | Chemical structure of 11 CSM5-K5.

These studies on naturally derived AMPs have collectively demonstrated their activity in inhibiting biofilm formation and expansion. However, natural AMPs appear less effective at eradicating established biofilms when administered alone or as part of co-treatment strategies. To overcome this, synthetic AMPs based on natural peptides have recently been engineered and trialled as biofilm eradication agents.

\section{Synthetic Antimicrobial Peptides}

Synthetic AMPs are peptides that have been synthesised de novo and engineered to have antimicrobial properties. As such, they have become a focus for many antibiotic co-treatment studies involving biofilms. Amongst the first synthetic peptides with confirmed antibacterial activities were those engineered by Kovacs et al., who reported their efficacy against Gram-positive and Gram-negative bacteria (Kovacs et al., 1960). Following that, several other synthetic AMPs have been designed and tested against a variety of bacterial pathogens. Synthetic peptide 1018 (amino acid sequence shown in Table 2) has been one studied extensively for its activity to potentiate antibiotics against biofilms. Reffuveille et al. initially tested the ability of 1018 to potentiate ciprofloxacin against $P$. aeruginosa biofilms (Reffuveille et al., 2014). Treating biofilms with ciprofloxacin alone at MIC, 10x MIC, and even 100x MIC had little eradication effect. However, combining peptide 1018 with ciprofloxacin, mostly eradicated $P$. aeruginosa biofilms and any remaining cells were small microcolonies of dead cells (Reffuveille et al., 2014). The efficacy of peptide 1018 in combination with other antibiotics was also tested against biofilms from multiple species (E. coli, S. aureus, Klebsiella pneumoniae, Acinetobacter baumannii, and Salmonella enterica) with combination treatment found always to be most effective when visually comparing biomass reduction and bacterial cell viability (Reffuveille et al., 2014). De la Fuente Núñez et al. also treated $P$. aeruginosa biofilms with the D-enantiomeric peptides DJK-5 and DJK-6 (DJK-6 amino acid sequence shown in Table 2) in combination with multiple antibiotics (ceftazidime, ciprofloxacin, imipenem, and tobramycin) (de la Fuente-Núñez et al., 2015). Using confocal microscopy and an in vivo C. elegans model, they showed that regardless of the combination, the concentration of antibiotic needed to inhibit biofilm growth was decreased at least 2-fold and in certain cases up to 16-fold with addition of the peptides (de la Fuente-Núñez et al., 2015). Similarly, DJK-6 potentiated meropenem against K. pneumoniae biofilms, reducing its MBEC value 16-fold, despite the fact that the tested isolates were carbapenemase producers (Ribeiro et al., 2015). Rudilla et al. also investigated combinations of synthetic peptides with imipenem, reporting that peptide AMP38 decreased by 8 -fold the imipenem $\mathrm{MBC}$ value for $P$. aeruginosa biofilms (Table 1) (Rudilla et al., 2016). Swedan et al. tested AMP WLBU2 in combination with imipenem, tobramycin, amoxicillin-clavulanate or ciprofloxacin against $K$. pneumoniae and A. baumannii biofilms, reporting that all combination treatments decreased MBEC values from 6- to 200-fold compared to treatment with WLBU2 alone, the dramatic 200fold improvement being in combination with ciprofloxacin against K. pneumoniae (Swedan et al., 2019). However, when testing cytotoxicity at MBEC values, a significant reduction in eukaryotic cell viability was observed for all tested concentrations of WLBU2 (Swedan et al., 2019). Due to their high cytotoxicity, WLBU2 combination treatment strategies might be more suitable for eradication of biofilms found in the environment e.g. on hospital surfaces or instruments. Alternatively, future efforts could be directed to modifying the chemical structure of WLBU2 to reduce cytotoxicity and facilitate the development of clinically viable antibiofilm agents.

In a more recent study, Pletzer et al. examined optimal combinations of synthetic AMPs with antibiotics to treat ESKAPE pathogen biofilms in an in vivo subcutaneous mouse abscess model (Pletzer et al., 2018). Mice infected with fluorescently tagged strains from the ESKAPE group and $E$. coli, inoculated at a dose of $\geq 10^{7}$ bacteria to simulate a chronic human wound, were monitored non-invasively for disease progression and treatment efficacy (Pletzer et al., 2018). 
Treatments were directly injected into the wound and were administered at approximate in vitro MIC concentrations (Pletzer et al., 2018). Antibiotic monotherapy even when administered at higher than MIC concentrations was often ineffective against high-density (biofilm) infections, highlighting that in vitro MICs are not reliably predictive of in vivo efficacy, which the authors also noted (Pletzer et al., 2018). Treatment with AMPs alone reduced wound size and moderately reduced bacterial numbers in the wound (2.2-22-fold decrease) (Pletzer et al., 2018). Notably, the AMP DJK-5 was very effective in combination with antibiotics (ciprofloxacin, gentamicin, meropenem, and vancomycin) in terms of reducing bacterial numbers in all species tested (ESKAPE and E. coli) (Pletzer et al., 2018).

While synthetic AMPs have not been studied as extensively as natural AMPs for antibiotic potentiation against biofilms, their promising potential has been clearly demonstrated. Their distinct advantages over naturally occurring AMPs - they can be readily improved via chemical manipulation, can be potentially linked to antibiotics, and engineered to be less toxic to mammalian cells- makes this class of antibiofilm agents very promising.

\section{Amino Acids}

Amino acids can exist in nature as a D-isomer or L-isomer, where the orientation of the alpha carbon in the molecule determines its chirality. The L-isomer is most common in ribosomal peptide synthesis, but recently $\mathrm{D}$-amino acids have also been found in mammals as regulators of neurogenesis and brain receptor function, and also components of some bacterial membranes (Cava et al., 2011). These have recently emerged as a class of potential biofilm dispersal agents given their role as regulators of biofilm dispersal (Cava et al., 2011). So far, the mode of action for D-amino acids has only been delineated in Bacillus subtilis biofilm dispersal, where the bacteria were reported to release D-leucine, -methionine, -tyrosine, and -tryptophan, which disrupted the amyloid fibers linking biofilm cells together at nanomolar concentrations (KolodkinGal et al., 2010).

While their exact mode of action has yet to be elucidated in other species, a few studies have been conducted using amino acids and antibiotics together as a biofilm treatment strategy. In one of these studies, Sanchez et al. investigated the ability of Damino acids and antibiotics to inhibit $P$. aeruginosa and S. aureus biofilm formation (Sanchez et al., 2014). Here, an equimolar mixture of D-amino acids (methionine, phenylalanine, and tryptophan) in combination with rifampicin were shown to reduce the minimum biofilm inhibition concentration (MBIC) by 4 -fold and a $>2$-log reduction in viable bacteria was reported with treated S. aureus biofilms (Sanchez et al., 2014). Similar results were observed for $P$. aeruginosa, with a $>2$-log reduction in viable bacteria when treated with $64 \mu \mathrm{g} / \mathrm{mL}$ rifampicin, and 5 $\mathrm{mM}$ of the D-amino acid mixture (Sanchez et al., 2014). Another study investigating amino acids in combination with antibiotics, conducted by Warraich et al., reported that compared to standalone treatment, lower concentrations of ciprofloxacin and D-amino acids were synergistic $(<40 \mathrm{mM}$ amino acids, $<90.54 \mu \mathrm{M}$ ciprofloxacin), and resulted in almost $97 \%$ inhibition of biofilm formation and $97.6 \%$ dispersal (D-isomer structures shown in Figure 7). However, biofilm eradication activity was not tested. The study also investigated the ability of L-amino acids to inhibit biofilm formation, and found that L-isomers of aspartic acid and glutamic acid were equally as effective as the D-isomers at $40 \mathrm{mM}$ (Warraich et al., 2020). Only two other studies have reported similar findings with L-amino acids (aspartate and glutamate) (Tong et al., 2014; Yang et al., 2015), challenging the dogma that L-isomers do not have antibiofilm activity. Conflicting evidence reports that L-amino acids can even encourage biofilm formation in some species (Hochbaum et al., 2011; Velmourougane and Prasanna, 2017).

For D-amino acids the evidence is more clear-cut, with several studies reporting some antibiofilm activity (inhibition and dispersal) and in a range of settings, from the industrial to development of drug delivery systems (Si et al., 2014; Wei et al., 2015; Li et al., 2016; Zilm et al., 2017). In these studies, D-amino acids (d-leucine, -methionine, -tyrosine, and -tryptophan) alone and in combination with other compounds effectively inhibited Desulfovibrio vulgaris biofilms and polymicrobial biofilms ( $\mathrm{Si}$ et al., 2014; Wei et al., 2015; Li et al., 2016; Zilm et al., 2017). $\mathrm{Li}$ et al. and $\mathrm{Si}$ et al. investigated disruption of established D. vulgaris biofilms that had been grown on carbon steel coupons (Li et al., 2016) or multi-species aggregates collected from an activated sludge reactor (Si et al., 2014) that had grown for longer than 10 days and up to 6 months (Si et al., 2014; Li et al., 2016). Both studies reported that combinations of D-amino acids with other compounds (hydroxymethyl phosphonium sulfate (THPS) and norspermidine) disrupted biofilm surface attachment (Si et al., 2014; Li et al., 2016). Titanium oxide<smiles>N[C@@H](CC(=O)O)C(=O)O</smiles>

12<smiles>NC(CCC(=O)O)C(=O)O</smiles>

FIGURE 7 | Chemical structures of 12 D-aspartic acid and 13 D-glutamic acid. 
nanoparticles engineered to release $\mathrm{D}$-amino acids upon stimulation with UV light were also shown effective in dispersing B. subtilis biofilms (Wei et al., 2015), and D-amino acids were also found to inhibit E. faecalis biofilm formation in vitro (Zilm et al., 2017). Some conflicting evidence exists around the capacity of $\mathrm{D}$-amino acids to disperse $P$. aeruginosa and S. aureus biofilms (Sarkar and Pires, 2015; Kao et al., 2017). Kao et al. investigated the ability of D-amino acids alanine, leucine, methionine, tryptophan, and tyrosine $(10 \mu \mathrm{M}, 1 \mathrm{mM}$, and $10 \mathrm{mM})$ to inhibit biofilm formation of $P$. aeruginosa strains PAO1 and PA14. Here they concluded that D-amino acids slow biofilm growth but do not prevent its formation (Kao et al., 2017), which Sarkar et al. also report for S. aureus biofilms treated with D-tryptophan and D-tyrosine at 1 and $5 \mathrm{mM}$ (Sarkar and Pires, 2015).

Several questions remain unanswered about the antibiofilm properties of amino acids and thus their potential to be employed in future antibiofilm strategies needs more fundamental research. This should aim to resolve the role of both $\mathrm{D}$ - and L- isomers in biofilm inhibition and dispersal and explore the specificity of their activity in key biofilm-producing species, such as $P$. aeruginosa and $S$. aureus. Their mode of action is likely to vary between species, and so, future research on this group of potential antibiofilm agents should be prioritized.

\section{REPURPOSED DRUGS}

Drug repurposing is a common strategy used in the field of medicine to maximise the potential of any individual drug for broader clinical applications outside of its original use (Farha and Brown, 2019). A small number of U.S. Food and Drug Administration (FDA)-approved drugs have been investigated against biofilms to date, particularly drugs involved in mucous degradation. $\mathrm{N}$-acetyl cysteine (structure shown in Figure 8) is a drug commonly prescribed to cystic fibrosis patients to break down mucus in the lung (Samuni et al., 2013). Moon et al. investigated $\mathrm{N}$-acetyl cysteine in combination with several antibiotics for dispersal of Prevotella intermedia biofilms, a major oral pathogen (Moon et al., 2015). They demonstrated that $\mathrm{N}$-acetyl cysteine was highly effective in preventing biofilm growth but was ineffective against pre-formed biofilms in vitro (Moon et al., 2015). Zhang et al. also investigated the efficacy of ambroxol (another FDA-approved drug for mucus degradation) in combination with vancomycin to treat Staphylococcus epidermidis biofilms in vitro and in vivo (Zhang et al., 2015). Interestingly, they reported that the combination of ambroxol and vancomycin was highly effective at eradicating mature biofilms in a rabbit intravenous catheter model, reducing catheter bacterial load by an impressive 7 logs compared to the control group (Table 1), which were only treated with heparin (Zhang et al., 2015). This result is very promising, and this combination should be further investigated with other bacterial species and other FDAapproved drugs used for mucus degradation.

Another FDA-approved drug with promising antibiofilm activity is auranofin. This gold-containing compound is usually prescribed for the treatment of rheumatoid arthritis, but has since been tested in combination with antibiotics against $S$. aureus and E. faecalis biofilms both in vitro and in vivo (She et al., 2019). Auranofin and the antibiotics fosfomycin, linezolid and chloramphenicol were synergistic at eradicating these biofilms in vitro, with compound combinations reducing individual $\mathrm{MBEC}_{50}$ values by 2- to over 8-fold (She et al., 2019). In vivo, combination treatment was reported to be similarly effective. In a $S$. aureus cutaneous infection mouse model, individual treatment with either auranofin, fosfomycin or linezolid resulted in a $<2-\log$ reduction in viable bacterial numbers, while combination treatment with an antibiotic and auranofin resulted in $>3-\log$ reduction in $S$. aureus CFU (She et al., 2019). In the same mouse model, however, treatment of E. faecalis infection was not reduced by more than 1-log in viable CFU irrespective of the treatment strategy, indicating that E. faecalis may have intrinsic resistance to auranofin both planktonically and in sessile form in vivo, despite the drug having activity in vitro (She et al., 2019).

While the potential use of FDA-approved drugs against biofilms is supported by some impressive results to date, evidence remains sporadic and additional studies are warranted. It would be interesting to investigate other existing mucolytic drugs, as well as approved anticoagulant/ antithrombotic and expectorant agents already approved for clinical use.

\section{CONCLUSIONS}

The combination of biofilm dispersal agents and antibiotics are an effective treatment strategy for both the prevention and eradication of bacterial biofilms. Many of these combinations have been successfully tested in vitro, and some also in vivo. All studies reviewed here have focused on single-species biofilms, however multi-species biofilms are also a major clinical and industrial issue (Del Pozo, 2018; Vishwakarma, 2020; Rather et al., 2021). Very few studies have been conducted investigating the dispersal of multi-species biofilms (Mei et al., 2013; Oliveira et al., 2014; Si et al., 2015; Wu et al., 2016; Ioannidis et al., 2019), and none featuring co-treatments with antibiotics. Multi-species biofilms are classically harder to eradicate than single-species biofilms given their heterogeneous composition, and research into the molecular interplay between participating species should illuminate potential therapeutic avenues (single or combination approaches) and advance the biofilm field going forward.

In most of these studies co-treatment or hybrid compounds have been much more effective in terms of biofilm inhibition and/or eradication than standalone treatments, with either the antibiofilm agent or antibiotic alone. This trend is evident across all classes of dispersal agents, suggesting that a two-pronged approach of biofilm dispersal and eradication could translate to a successful treatment strategy. Furthermore, there appears to be a distinct advantage to the simultaneous delivery and or release of the two agents at the target site, an effect which was demonstrated by the production of hybrid molecules, and delivery and release systems. However, very 


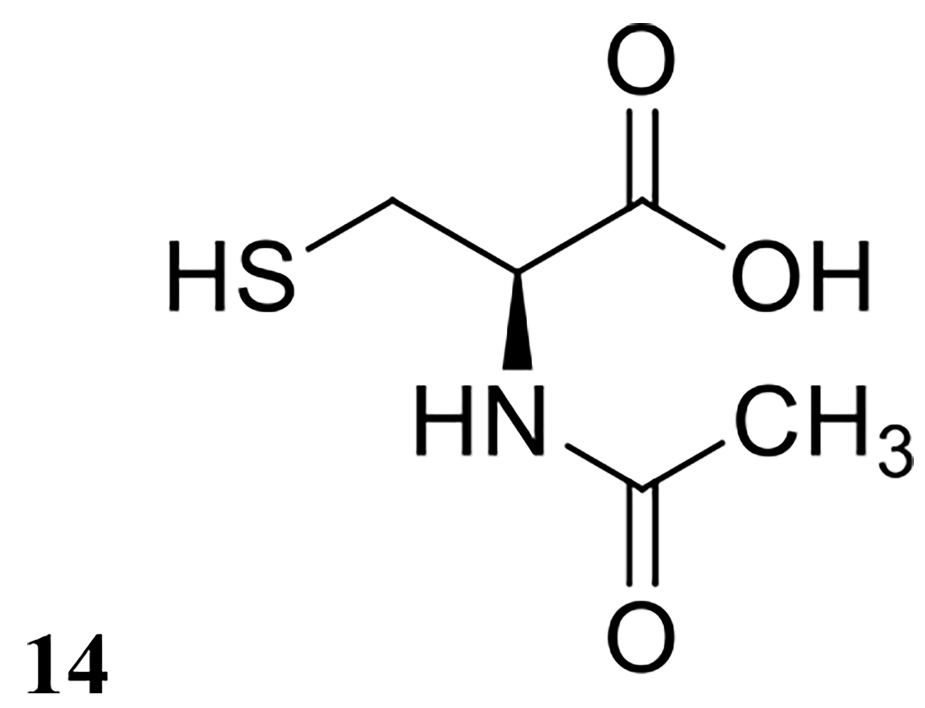

FIGURE 8 | Chemical structure of $14 \mathrm{~N}$-acetyl cysteine.

few cotreatments have been explored for their potential as hybrids drugs. This review has highlighted a multitude of effective cotreatments that would be ideal for future development into hybrid drugs.

Despite the advantages of some combination strategies having been demonstrated both in vitro and in vivo, follow-up in vivo studies are often lacking for co-treatments with confirmed antibiofilm activity in vitro. Indeed, out of the 45 studies reviewed, only $17(\sim 38 \%)$ progressed from in vitro testing to preclinical evaluation in an in vivo model. Furthermore, out of the hundreds of newly discovered antibiofilm agents currently reported in the literature, only a handful have been tested for activity in vitro in assays comparing single agents over co-treatment. This highlights that there is still a lot of untapped potential for the development of new effective anti-biofilm combination therapies. Future studies on novel antibiofilm agents should follow a cotreatment design from early testing, first examining the agent's antibiotic potentiation activity in vitro and if successful progress into in vivo testing in relevant animal models. While this process can appear tedious, it is necessary in order to shortlist promising combination leads for clinical development. We posit that this review has provided examples that are already following this process successfully and identified others that represent promising candidates to follow this path.

Of the agents reviewed here, QSIs, NO, and nitroxides appear to hold the most promise for the development of biofilmeradication treatments based on the co-treatment strategy. While the efficacy of co-treatments using QSIs has been demonstrated in vivo in some species, both $\mathrm{NO}$ and nitroxides remain to be examined in vivo despite their promising in vitro results. Any of these candidates would be ideal to examine in in vivo models.

Biofilm-related infection remains a critical healthcare issue worldwide, and new and innovative strategies need to be devised. Co-treatment using antibiofilm agents with antibiotics appears to hold great promise as one such strategy, and our review provides useful information required to progress and assist in the development of candidates along this strategy.

\section{AUTHOR CONTRIBUTIONS}

AV and MT contributed to conception and design of the review. $\mathrm{SH}$ and $\mathrm{AV}$ compiled and reviewed relevant studies. $\mathrm{SH}$ wrote the first draft of the manuscript, figures, and tables. AV and MT wrote sections of the manuscript and edited tables. All authors contributed to the article and approved the submitted version.

\section{FUNDING}

SH is the recipient of an Australian Government Research Training Program (RTP) Scholarship.

\section{ACKNOWLEDGMENTS}

MT gratefully acknowledges funding support from the Australian Research Council (Discovery Projects DP190101613 and DP210101317, and a Georgina Sweet Award for Women in Quantitative Biomedical Science), the National Health and Medical Research Council of Australia (Project Grant GNT1144046), and the Clive and Vera Ramaciotti Foundations (Health Investment Grant 2017HIG0119).

\section{SUPPLEMENTARY MATERIAL}

The Supplementary Material for this article can be found online at: https://www.frontiersin.org/articles/10.3389/fcimb.2022. 850030/full\#supplementary-material 


\section{REFERENCES}

Ammons, M. C., and Copié, V. (2013). Mini-Review: Lactoferrin: A Bioinspired, Anti-Biofilm Therapeutic. Biofouling 29 (4), 443-455. doi: 10.1080/ 08927014.2013.773317

Anjum, A., Sim, C. H., and Ng, S. F. (2018). Hydrogels Containing Antibiofilm and Antimicrobial Agents Beneficial for Biofilm-Associated Wound Infection: Formulation Characterizations and In Vitro Study. AAPS PharmSciTech 19 (3), 1219-1230. doi: 10.1208/s12249-017-0937-4

Azevedo, M. M., Lisboa, C., Cobrado, L., Pina-Vaz, C., and Rodrigues, A. (2020). Hard-To-Heal Wounds, Biofilm and Wound Healing: An Intricate Interrelationship. Br. J. Nurs. 29 (5), S6-s13. doi: 10.12968/bjon.2020.29.5.S6

Barnes, R. J., Bandi, R. R., Wong, W. S., Barraud, N., McDougald, D., Fane, A., et al. (2013). Optimal Dosing Regimen of Nitric Oxide Donor Compounds for the Reduction of Pseudomonas Aeruginosa Biofilm and Isolates From Wastewater Membranes. Biofouling 29 (2), 203-212. doi: 10.1080/ 08927014.2012.760069

Barraud, N., Hassett, D. J., Hwang, S. H., Rice, S. A., Kjelleberg, S., and Webb, J. S. (2006). Involvement of Nitric Oxide in Biofilm Dispersal of Pseudomonas Aeruginosa. J. Bacteriol. 188 (21), 7344-7353. doi: 10.1128/JB.00779-06

Barraud, N., Kardak, B. G., Yepuri, N. R., Howlin, R. P., Webb, J. S., Faust, S. N., et al. (2012). Cephalosporin-3'-Diazeniumdiolates: Targeted NO-Donor Prodrugs for Dispersing Bacterial Biofilms. Angew Chem. Int. Ed. Engl. 51 (36), 9057-9060. doi: 10.1002/anie.201202414

Barraud, N., Kelso, M. J., Rice, S. A., and Kjelleberg, S. (2015). Nitric Oxide: A Key Mediator of Biofilm Dispersal With Applications in Infectious Diseases. Curr. Pharm. Des. 21 (1), 31-42. doi: 10.2174/1381612820666140905112822

Barraud, N., Storey, M. V., Moore, Z. P., Webb, J. S., Rice, S. A., and Kjelleberg, S. (2009). Nitric Oxide-Mediated Dispersal in Single- and Multi-Species Biofilms of Clinically and Industrially Relevant Microorganisms. Microb. Biotechnol. 2 (3), 370-378. doi: 10.1111/j.1751-7915.2009.00098.x

Bialvaei, A. Z., and Samadi Kafil, H. (2015). Colistin, Mechanisms and Prevalence of Resistance. Curr. Med. Res. Opin. 31 (4), 707-721. doi: 10.1185/ 03007995.2015.1018989

Brackman, G., Breyne, K., De Rycke, R., Vermote, A., Van Nieuwerburgh, F., Meyer, E., et al. (2016). The Quorum Sensing Inhibitor Hamamelitannin Increases Antibiotic Susceptibility of Staphylococcus Aureus Biofilms by Affecting Peptidoglycan Biosynthesis and eDNA Release. Sci. Rep. 6, 20321. doi: $10.1038 /$ srep 20321

Brackman, G., and Coenye, T. (2015). Quorum Sensing Inhibitors as Anti-Biofilm Agents. Curr. Pharm. Des. 21 (1), 5-11. doi: 10.2174/13816128206 66140905114627

Brackman, G., Cos, P., Maes, L., Nelis, H. J., and Coenye, T. (2011). Quorum Sensing Inhibitors Increase the Susceptibility of Bacterial Biofilms to Antibiotics In Vitro and In Vivo. Antimicrob. Agents Chemother. 55 (6), 2655-2661. doi: 10.1128/AAC.00045-11

Brackman, G., Garcia-Fernandez, M. J., Lenoir, J., De Meyer, L., Remon, J. P., De Beer, T., et al. (2016). Dressings Loaded With Cyclodextrin-Hamamelitannin Complexes Increase Staphylococcus Aureus Susceptibility Toward Antibiotics Both in Single as Well as in Mixed Biofilm Communities. Macromol. Biosci. 16 (6), 859-869. doi: 10.1002/mabi.201500437

Cava, F., Lam, H., de Pedro, M. A., and Waldor, M. K. (2011). Emerging Knowledge of Regulatory Roles of D-Amino Acids in Bacteria. Cell Mol. Life Sci. 68 (5), 817-831. doi: 10.1007/s00018-010-0571-8

Cheng, W. J., Zhou, J. W., Zhang, P. P., Luo, H. Z., Tang, S., Li, J. J., et al. (2020). Quorum Sensing Inhibition and Tobramycin Acceleration in Chromobacterium Violaceum by Two Natural Cinnamic Acid Derivatives. Appl. Microbiol. Biotechnol. 104 (11), 5025-5037. doi: 10.1007/s00253-02010593-0

Christensen, L. D., van Gennip, M., Jakobsen, T. H., Alhede, M., Hougen, H. P., Høiby, N., et al. (2012). Synergistic Antibacterial Efficacy of Early Combination Treatment With Tobramycin and Quorum-Sensing Inhibitors Against Pseudomonas Aeruginosa in an Intraperitoneal Foreign-Body Infection Mouse Model. J. Antimicrob. Chemother. 67 (5), 1198-1206. doi: 10.1093/ $\mathrm{jac} / \mathrm{dks} 002$

Cirioni, O., Mocchegiani, F., Cacciatore, I., Vecchiet, J., Silvestri, C., Baldassarre, L., et al. (2013). Quorum Sensing Inhibitor FS3-Coated Vascular Graft
Enhances Daptomycin Efficacy in a Rat Model of Staphylococcal Infection. Peptides 40, 77-81. doi: 10.1016/j.peptides.2012.12.002

Cui, L., H-m, N., Shoji, M., and Hiramatsu, K. (2009). Contribution of vraSR and graSR Point Mutations to Vancomycin Resistance in VancomycinIntermediate Staphylococcus Aureus. Antimicrob Agents Chemother. 53 (3), 1231-1234. doi: 10.1128/AAC.01173-08

Davies, K. M., Wink, D. A., Saavedra, J. E., and Keefer, L. K. (2001). Chemistry of the Diazeniumdiolates. 2. Kinetics and Mechanism of Dissociation to Nitric Oxide in Aqueous Solution. J. Am. Chem. Soc. 123 (23), 5473-5481. doi: $10.1021 / \mathrm{ja} 002899 \mathrm{q}$

de la Fuente-Núñez, C., Reffuveille, F., Fairfull-Smith, K. E., and Hancock, R. E. (2013). Effect of Nitroxides on Swarming Motility and Biofilm Formation, Multicellular Behaviors in Pseudomonas Aeruginosa. Antimicrob. Agents Chemother. 57 (10), 4877-4881. doi: 10.1128/AAC.01381-13

de la Fuente-Núñez, C., Reffuveille, F., Mansour, S. C., Reckseidler-Zenteno, S. L., Hernández, D., Brackman, G., et al. (2015). D-Enantiomeric Peptides That Eradicate Wild-Type and Multidrug-Resistant Biofilms and Protect Against Lethal Pseudomonas Aeruginosa Infections. Chem. Biol. 22 (2), 196-205. doi: 10.1016/j.chembiol.2015.01.002

Del Pozo, J. L. (2018). Biofilm-Related Disease. Expert Rev. Anti Infect. Ther. 16 (1), 51-65. doi: 10.1080/14787210.2018.1417036

Deppisch, C., Herrmann, G., Graepler-Mainka, U., Wirtz, H., Heyder, S., Engel, C. et al. (2016). Gaseous Nitric Oxide to Treat Antibiotic Resistant Bacterial and Fungal Lung Infections in Patients With Cystic Fibrosis: A Phase I Clinical Study. Infection 44 (4), 513-520. doi: 10.1007/s15010-016-0879-x

Eckert, R., Brady, K. M., Greenberg, E. P., Qi, F., Yarbrough, D. K., He, J., et al. (2006). Enhancement of Antimicrobial Activity Against Pseudomonas Aeruginosa by Coadministration of G10KHc and Tobramycin. Antimicrob. Agents Chemother. 50 (11), 3833-3838. doi: 10.1128/AAC.00509-06

Farha, M. A., and Brown, E. D. (2019). Drug Repurposing for Antimicrobial Discovery. Nat. Microbiol. 4 (4), 565-577. doi: 10.1038/s41564-019-0357-1

Field, D., Seisling, N., Cotter, P. D., Ross, R. P., and Hill, C. (2016). Synergistic Nisin-Polymyxin Combinations for the Control of Pseudomonas Biofilm Formation. Front. Microbiol. 7, 1713. doi: 10.3389/fmicb.2016.01713

Findlay, V. J., Townsend, D. M., Saavedra, J. E., Buzard, G. S., Citro, M. L., Keefer, L. K., et al. (2004). Tumor Cell Responses to a Novel Glutathione STransferase-Activated Nitric Oxide-Releasing Prodrug. Mol. Pharmacol. 65 (5), 1070. doi: 10.1124/mol.65.5.1070

Fleming, D., and Rumbaugh, K. P. (2017). Approaches to Dispersing Medical Biofilms. Microorganisms 5 (2), 15. doi: 10.3390/microorganisms5020015

Fleming, D., and Rumbaugh, K. (2018). The Consequences of Biofilm Dispersal on the Host. Sci. Rep. 8 (1), 10738. doi: 10.1038/s41598-018-29121-2

Furiga, A., Lajoie, B., El Hage, S., Baziard, G., and Roques, C. (2015). Impairment of Pseudomonas Aeruginosa Biofilm Resistance to Antibiotics by Combining the Drugs With a New Quorum-Sensing Inhibitor. Antimicrob. Agents Chemother. 60 (3), 1676-1686. doi: 10.1128/AAC.02533-15

Ghosh, A., Jayaraman, N., and Chatterii, D. (2020). Small-Molecule Inhibition of Bacterial Biofilm. ACS Omega 5 (7), 3108-3115. doi: 10.1021/acsomega. 9b03695

Gilbert, P., Maira-Litran, T., McBain, A. J., Rickard, A. H., and Whyte, F. W. (2002). The Physiology and Collective Recalcitrance of Microbial Biofilm Communities. Adv. Microb. Physiol. 46, 202-256. doi: 10.1016/S0065-2911(02)46005-5

Grassi, L., Maisetta, G., Esin, S., and Batoni, G. (2017). Combination Strategies to Enhance the Efficacy of Antimicrobial Peptides Against Bacterial Biofilms. Front. Microbiol. 8, 2409. doi: 10.3389/fmicb.2017.02409

Høiby, N., Bjarnsholt, T., Givskov, M., Molin, S., and Ciofu, O. (2010). Antibiotic Resistance of Bacterial Biofilms. Int. J. Antimicrob. Agents 35 (4), 322-332. doi: 10.1016/j.ijantimicag.2009.12.011

Hall, M. J., Middleton, R. F., and Westmacott, D. (1983). The Fractional Inhibitory Concentration (FIC) Index as a Measure of Synergy. J. Antimicrob. Chemother. 11 (5), 427-433. doi: 10.1093/jac/11.5.427

Hochbaum, A. I., Kolodkin-Gal, I., Foulston, L., Kolter, R., Aizenberg, J., and Losick, R. (2011). Inhibitory Effects of D-Amino Acids on Staphylococcus Aureus Biofilm Development. J. Bacteriol 193 (20), 5616-5622. doi: 10.1128/JB.05534-11

Ho, D. K., Murgia, X., De Rossi, C., Christmann, R., Hüfner de Mello Martins, A. G., Koch, M., et al. (2020). Squalenyl Hydrogen Sulfate Nanoparticles for Simultaneous Delivery of Tobramycin and an Alkylquinolone Quorum 
Sensing Inhibitor Enable the Eradication of P aeruginosa Biofilm Infect. Angew Chem. Int. Ed Engl. 59 (26), 10292-10296. doi: 10.1002/anie.202001407

Hossain, S., and Boon, E. M. (2017). Discovery of a Novel Nitric Oxide Binding Protein and Nitric-Oxide-Responsive Signaling Pathway in Pseudomonas Aeruginosa. ACS Infect. Dis. 3 (6), 454-461. doi: 10.1021/acsinfecdis.7b00027

Hossain, S., Nisbett, L. M., and Boon, E. M. (2017). Discovery of Two Bacterial Nitric Oxide-Responsive Proteins and Their Roles in Bacterial Biofilm Regulation. Acc Chem. Res. 50 (7), 1633-1639. doi: 10.1021/acs.accounts.7b00095

Ioannidis, K., Niazi, S., Mylonas, P., Mannocci, F., and Deb, S. (2019). The Synthesis of Nano Silver-Graphene Oxide System and its Efficacy Against Endodontic Biofilms Using a Novel Tooth Model. Dent. Mater. 35 (11), 16141629. doi: $10.1016 /$ j.dental.2019.08.105

Jiang, Q., Chen, J., Yang, C., Yin, Y., and Yao, K. (2019). Quorum Sensing: A Prospective Therapeutic Target for Bacterial Diseases. BioMed. Res. Int. 2019, 2015978. doi: 10.1155/2019/2015978

Jorge, P., Grzywacz, D., Kamysz, W., Lourenço, A., and Pereira, M. O. (2017). Searching for New Strategies Against Biofilm Infections: Colistin-AMP Combinations Against Pseudomonas Aeruginosa and Staphylococcus Aureus Single- and Double-Species Biofilms. PloS One 12 (3), e0174654. doi: 10.1371/ journal.pone. 0174654

Kalsy, M., Tonk, M., Hardt, M., Dobrindt, U., Zdybicka-Barabas, A., Cytrynska, M., et al. (2020). The Insect Antimicrobial Peptide Cecropin A Disrupts Uropathogenic Escherichia Coli Biofilms. NPJ Biofilms Microbiomes 6 (1), 6. doi: 10.1038/s41522-020-0116-3

Kao, W. T., Frye, M., Gagnon, P., Vogel, J. P., and Chole, R. (2017). D-Amino Acids do Not Inhibit Pseudomonas Aeruginosa Biofilm Formation. Laryngoscope Investig. Otolaryngol. 2 (1), 4-9. doi: 10.1002/lio2.34

Kaplan, J. B. (2010). Biofilm Dispersal: Mechanisms, Clinical Implications, and Potential Therapeutic Uses. J. Dent. Res. 89 (3), 205-218. doi: 10.1177/ 0022034509359403

Kolodkin-Gal, I., Romero, D., Cao, S., Clardy, J., Kolter, R., and Losick, R. (2010). D-Amino Acids Trigger Biofilm Disassembly. Science 328 (5978), 627-629. doi: $10.1126 /$ science. 1188628

Kovacs, K., Kotai, A., and Szabo, I. (1960). Synthetic Polypeptide Derivatives With Anti-Bacterial Activities. Nature 185, 266-267. doi: 10.1038/185266a0

Krishan, A. (1975). Rapid Flow Cytofluorometric Analysis of Mammalian Cell Cycle by Propidium Iodide Staining. J. Cell Biol. 66 (1), 188-193. doi: 10.1083/ jcb.66.1.188

Lachica, M., Anutrakunchai, C., Prajaneh, S., Nazmi, K., Bolscher, J. G. M., and Taweechaisupapong, S. (2019). Synergistic Effects of LFchimera and Antibiotic Against Planktonic and Biofilm Form of Aggregatibacter Actinomycetemcomitans. PloS One 14 (7), e0217205. doi: 10.1371/journal. pone. 0217205

Lewis, K. (2008). Multidrug Tolerance of Biofilms and Persister Cells. Curr. Top. Microbiol. Immunol. 322, 107-131. doi: 10.1007/978-3-540-75418-3_6

Li, Y., Jia, R., Al-Mahamedh, H. H., Xu, D., and Gu, T. (2016). Enhanced Biocide Mitigation of Field Biofilm Consortia by a Mixture of D-Amino Acids. Front. Microbiol. 7, 896. doi: 10.3389/fmicb.2016.00896

Liu, S., Cai, X., Xue, W., Ma, D., and Zhang, W. (2020). Chitosan Derivatives CoDelivering Nitric Oxide and Methicillin for the Effective Therapy to the Methicillin-Resistant S. aureus Infection. Carbohydr Polym 234, 115928. doi: 10.1016/j.carbpol.2020.115928

Luo, J., Dong, B., Wang, K., Cai, S., Liu, T., Cheng, X., et al. (2017). Baicalin Inhibits Biofilm Formation, Attenuates the Quorum Sensing-Controlled Virulence and Enhances Pseudomonas Aeruginosa Clearance in a Mouse Peritoneal Implant Infection Model. PloS One 12 (4), e0176883. doi: 10.1371/journal.pone.0176883

Lu, Y., Slomberg, D. L., Shah, A., and Schoenfisch, M. H. (2013). Nitric OxideReleasing Amphiphilic Poly(Amidoamine) (PAMAM) Dendrimers as Antibacterial Agents. Biomacromolecules 14 (10), 3589-3598. doi: 10.1021/ bm400961r

Maiden, M. M., Zachos, M. P., and Waters, C. M. (2019). Hydrogels Embedded With Melittin and Tobramycin Are Effective Against Pseudomonas Aeruginosa Biofilms in an Animal Wound Model. Front. Microbiol. 10, 1348. doi: $10.3389 /$ fmicb. 2019.01348

Marvasi, M., Durie, I. A., McLamore, E. S., Vanegas, D. C., and Chaturvedi, P. (2015). Salmonella Enterica Biofilm-Mediated Dispersal by Nitric Oxide
Donors in Association With Cellulose Nanocrystal Hydrogels. AMB Express 5, 28. doi: 10.1186/s13568-015-0114-7

Mataraci, E., and Dosler, S. (2012). In Vitro Activities of Antibiotics and Antimicrobial Cationic Peptides Alone and in Combination Against Methicillin-Resistant Staphylococcus Aureus Biofilms. Antimicrob. Agents Chemother. 56 (12), 6366-6371. doi: 10.1128/AAC.01180-12

McDougald, D., Rice, S. A., Barraud, N., Steinberg, P. D., and Kjelleberg, S. (2011). Should We Stay or Should We Go: Mechanisms and Ecological Consequences for Biofilm Dispersal. Nat. Rev. Microbiol. 10 (1), 39-50. doi: 10.1038/ nrmicro2695

Mei, M. L., Li, Q. L., Chu, C. H., Lo, E. C., and Samaranayake, L. P. (2013). Antibacterial Effects of Silver Diamine Fluoride on Multi-Species Cariogenic Biofilm on Caries. Ann. Clin. Microbiol. Antimicrob. 12, 4. doi: 10.1186/14760711-12-4

Miller, C. C., Hergott, C. A., Rohan, M., Arsenault-Mehta, K., Döring, G., and Mehta, S. (2013). Inhaled Nitric Oxide Decreases the Bacterial Load in a Rat Model of Pseudomonas Aeruginosa Pneumonia. J. Cyst Fibros 12 (6), 817-820. doi: $10.1016 /$ j.jcf.2013.01.008

Miller, C., Miller, M., McMullin, B., Regev, G., Serghides, L., Kain, K., et al. (2012). A Phase I Clinical Study of Inhaled Nitric Oxide in Healthy Adults. J. Cyst Fibros 11 (4), 324-331. doi: 10.1016/j.jcf.2012.01.003

Minardi, D., Ghiselli, R., Cirioni, O., Giacometti, A., Kamysz, W., Orlando, F., et al. (2007). The Antimicrobial Peptide Tachyplesin III Coated Alone and in Combination With Intraperitoneal Piperacillin-Tazobactam Prevents Ureteral Stent Pseudomonas Infection in a Rat Subcutaneous Pouch Model. Peptides 28 (12), 2293-2298. doi: 10.1016/j.peptides.2007.10.001

Moon, J. H., Jang, E. Y., Shim, K. S., and Lee, J. Y. (2015). In Vitro Effects of NAcetyl Cysteine Alone and in Combination With Antibiotics on Prevotella Intermedia. J. Microbiol. 53 (5), 321-329. doi: 10.1007/s12275-015-4500-2

Müsken, M., Pawar, V., Schwebs, T., Bähre, H., Felgner, S., Weiss, S., et al. (2018). Breaking the Vicious Cycle of Antibiotic Killing and Regrowth of BiofilmResiding Pseudomonas Aeruginosa. Antimicrob. Agents Chemother. 62 (12), e01635-18. doi: 10.1128/AAC.01635-18

Nguyen, T. K., Selvanayagam, R., Ho, K. K. K., Chen, R., Kutty, S. K., Rice, S. A., et al. (2016). Co-Delivery of Nitric Oxide and Antibiotic Using Polymeric Nanoparticles. Chem. Sci. 7 (2), 1016-1027. doi: 10.1039/C5SC02769A

Nisbett, L. M., Binnenkade, L., Bacon, B., Hossain, S., Kotloski, N. J., Brutinel, E. D., et al. (2019). NosP Signaling Modulates the NO/H-NOX-Mediated Multicomponent C-Di-GMP Network and Biofilm Formation in Shewanella Oneidensis. Biochemistry 58 (48), 4827-4841. doi: 10.1021/acs. biochem.9b00706

O'Loughlin, C. T., Miller, L. C., Siryaporn, A., Drescher, K., Semmelhack, M. F., and Bassler, B. L. (2013). A Quorum-Sensing Inhibitor Blocks Pseudomonas Aeruginosa Virulence and Biofilm Formation. Proc. Natl. Acad. Sci. U. S. A. 110 (44), 17981-17986. doi: 10.1073/pnas.1316981110

Oliveira, S. A., Zambrana, J. R., Iorio, F. B., Pereira, C. A., and Jorge, A. O. (2014). The Antimicrobial Effects of Citrus Limonum and Citrus Aurantium Essential Oils on Multi-Species Biofilms. Braz. Oral. Res. 28, 22-27. doi: 10.1590/S180683242013005000024

Orlando, F., Ghiselli, R., Cirioni, O., Minardi, D., Tomasinsig, L., Mocchegiani, F., et al. (2008). BMAP-28 Improves the Efficacy of Vancomycin in Rat Models of Gram-Positive Cocci Ureteral Stent Infection. Peptides 29 (7), 1118-1123. doi: 10.1016/j.peptides.2008.03.005

Pletzer, D., and Hancock, R. E. (2016). Antibiofilm Peptides: Potential as BroadSpectrum Agents. J. Bacteriol. 198 (19), 2572-2578. doi: 10.1128/JB.00017-16

Pletzer, D., Mansour, S. C., and Hancock, R. E. W. (2018). Synergy Between Conventional Antibiotics and Anti-Biofilm Peptides in a Murine, SubCutaneous Abscess Model Caused by Recalcitrant ESKAPE Pathogens. PloS Pathog. 14 (6), e1007084. doi: 10.1371/journal.ppat.1007084

Poh, W. H., Barraud, N., Guglielmo, S., Lazzarato, L., Rolando, B., Fruttero, R., et al. (2017). Furoxan Nitric Oxide Donors Disperse Pseudomonas Aeruginosa Biofilms, Accelerate Growth, and Repress Pyoverdine Production. ACS Chem. Biol. 12 (8), 2097-2106. doi: 10.1021/acschembio.7b00256

Rajasekaran, G., Dinesh Kumar, S., Nam, J., Jeon, D., Kim, Y., Lee, C. W., et al. (2019). Antimicrobial and Anti-Inflammatory Activities of Chemokine CXCL14-Derived Antimicrobial Peptide and its Analogs. Biochim. Biophys. Acta Biomembr. 1861 (1), 256-267. doi: 10.1016/j.bbamem.2018.06.016 
Rather, M. A., Gupta, K., Bardhan, P., Borah, M., Sarkar, A., Eldiehy, K. S. H., et al. (2021). Microbial Biofilm: A Matter of Grave Concern for Human Health and Food Industry. J. Basic Microbiol. 61 (5), 380-395. doi: 10.1002/jobm.202000678

Reffuveille, F., de la Fuente-Núñez, C., Mansour, S., and Hancock, R. E. (2014). A Broad-Spectrum Antibiofilm Peptide Enhances Antibiotic Action Against Bacterial Biofilms. Antimicrob. Agents Chemother. 58 (9), 5363-5371. doi: 10.1128/AAC.03163-14

Reffuveille, F., Fuente-Núñez Cde, L., Fairfull-Smith, K. E., and Hancock, R. E. (2015). Potentiation of Ciprofloxacin Action Against Gram-Negative Bacterial Biofilms by a Nitroxide. Pathog. Dis. 73 (5), ftv016. doi: 10.1093/femspd/ftv016

Ren, H., Wu, J., Colletta, A., Meyerhoff, M. E., and Xi, C. (2016). Efficient Eradication of Mature Pseudomonas Aeruginosa Biofilm via Controlled Delivery of Nitric Oxide Combined With Antimicrobial Peptide and Antibiotics. Front. Microbiol. 7, 1260. doi: 10.3389/fmicb.2016.01260

Ribeiro, S. M., de la Fuente-Núñez, C., Baquir, B., Faria-Junior, C., Franco, O. L., and Hancock, R. E. (2015). Antibiofilm Peptides Increase the Susceptibility of Carbapenemase-Producing Klebsiella Pneumoniae Clinical Isolates to $\beta$ Lactam Antibiotics. Antimicrob. Agents Chemother. 59 (7), 3906-3912. doi: 10.1128/AAC.00092-15

Roy, R., Tiwari, M., Donelli, G., and Tiwari, V. (2018). Strategies for Combating Bacterial Biofilms: A Focus on Anti-Biofilm Agents and Their Mechanisms of Action. Virulence 9 (1), 522-554. doi: 10.1080/21505594.2017.1313372

Rudilla, H., Fusté, E., Cajal, Y., Rabanal, F., Vinuesa, T., and Viñas, M. (2016). Synergistic Antipseudomonal Effects of Synthetic Peptide AMP38 and Carbapenems. Molecules 21 (9), 1223. doi: 10.3390/molecules21091223

Sadowska-Bartosz, I., Gajewska, A., Skolimowski, J., Szewczyk, R., and Bartosz, G. (2015). Nitroxides Protect Against Peroxynitrite-Induced Nitration and Oxidation. Free Radic. Biol. Med. 89, 1165-1175. doi: 10.1016/ j.freeradbiomed.2015.11.002

Samuni, Y., Goldstein, S., Dean, O. M., and Berk, M. (2013). The Chemistry and Biological Activities of N-Acetylcysteine. Biochim. Biophys. Acta 1830 (8), 4117-4129. doi: 10.1016/j.bbagen.2013.04.016

Sanchez, C. J. Jr., Akers, K. S., Romano, D. R., Woodbury, R. L., Hardy, S. K., Murray, C. K., et al. (2014). D-Amino Acids Enhance the Activity of Antimicrobials Against Biofilms of Clinical Wound Isolates of Staphylococcus Aureus and Pseudomonas Aeruginosa. Antimicrob. Agents Chemother. 58 (8), 4353-4361. doi: 10.1128/AAC.02468-14

Sarkar, S., and Pires, M. M. (2015). D-Amino Acids do Not Inhibit Biofilm Formation in Staphylococcus Aureus. PloS One 10 (2), e0117613. doi: 10.1371/ journal.pone.0117613

Schairer, D. O., Chouake, J. S., Nosanchuk, J. D., and Friedman, A. J. (2012). The Potential of Nitric Oxide Releasing Therapies as Antimicrobial Agents. Virulence 3 (3), 271-279. doi: 10.4161/viru.20328

She, P., Zhou, L., Li, S., Liu, Y., Xu, L., Chen, L., et al. (2019). Synergistic Microbicidal Effect of Auranofin and Antibiotics Against Planktonic and Biofilm-Encased S. Aureus and E. Faecalis. Front. Microbiol. 10, 2453. doi: 10.3389/fmicb.2019.02453

Simonetti, O., Cirioni, O., Cacciatore, I., Baldassarre, L., Orlando, F., Pierpaoli, E., et al. (2016). Efficacy of the Quorum Sensing Inhibitor FS10 Alone and in Combination With Tigecycline in an Animal Model of Staphylococcal Infected Wound. PloS One 11 (6), e0151956. doi: 10.1371/journal.pone.0151956

Simonetti, O., Cirioni, O., Mocchegiani, F., Cacciatore, I., Silvestri, C., Baldassarre, L., et al. (2013). The Efficacy of the Quorum Sensing Inhibitor FS8 and Tigecycline in Preventing Prosthesis Biofilm in an Animal Model of Staphylococcal Infection. Int. J. Mol. Sci. 14 (8), 16321-16332. doi: 10.3390/ ijms 140816321

Singh, N., Romero, M., Travanut, A., Monteiro, P. F., Jordana-Lluch, E., Hardie, K. R., et al. (2019). Dual Bioresponsive Antibiotic and Quorum Sensing Inhibitor Combination Nanoparticles for Treatment of Pseudomonas Aeruginosa Biofilms In Vitro and Ex Vivo. Biomater. Sci. 7 (10), 4099-4111. doi: 10.1039/C9BM00773C

Si, X., Quan, X., Li, Q., and Wu, Y. (2014). Effects of D-Amino Acids and Norspermidine on the Disassembly of Large, Old-Aged Microbial Aggregates. Water Res. 54, 247-253. doi: 10.1016/j.watres.2014.02.007

Si, X., Quan, X., and Wu, Y. (2015). A Small-Molecule Norspermidine and Norspermidine-Hosting Polyelectrolyte Coatings Inhibit Biofilm Formation by Multi-Species Wastewater Culture. Appl. Microbiol. Biotechnol. 99 (24), 10861-10870. doi: 10.1007/s00253-015-6943-0
Slachmuylders, L., Van Acker, H., Brackman, G., Sass, A., Van Nieuwerburgh, F., and Coenye, T. (2018). Elucidation of the Mechanism Behind the Potentiating Activity of Baicalin Against Burkholderia Cenocepacia Biofilms. PloS One 13 (1), e0190533. doi: 10.1371/journal.pone.0190533

Soren, O., Rineh, A., Silva, D. G., Cai, Y., Howlin, R. P., Allan, R. N., et al. (2020). Cephalosporin Nitric Oxide-Donor Prodrug DEA-C3D Disperses Biofilms Formed by Clinical Cystic Fibrosis Isolates of Pseudomonas Aeruginosa. J. Antimicrob. Chemother. 75 (1), 117-125. doi: 10.1093/jac/dkz378

Swedan, S., Shubair, Z., and Almaaytah, A. (2019). Synergism of Cationic Antimicrobial Peptide WLBU2 With Antibacterial Agents Against Biofilms of Multi-Drug Resistant Acinetobacter Baumannii and Klebsiella Pneumoniae. Infect. Drug Resist. 12, 2019-2030. doi: 10.2147/IDR.S215084

Thappeta, K. R. V., Vikhe, Y. S., Yong, A. M. H., Chan-Park, M. B., and Kline, K. A. (2020). Combined Efficacy of an Antimicrobial Cationic Peptide Polymer With Conventional Antibiotics to Combat Multidrug-Resistant Pathogens. ACS Infect. Dis. 6 (5), 1228-1237. doi: 10.1021/acsinfecdis.0c00016

Tong, Z., Zhang, L., Ling, J., Jian, Y., Huang, L., and Deng, D. (2014). An In Vitro Study on the Effect of Free Amino Acids Alone or in Combination With Nisin on Biofilms as Well as on Planktonic Bacteria of Streptococcus Mutans. PloS One 9 (6), e99513. doi: 10.1371/journal.pone.0099513

Tong, Z., Zhang, Y., Ling, J., Ma, J., Huang, L., and Zhang, L. (2014). An In Vitro Study on the Effects of Nisin on the Antibacterial Activities of 18 Antibiotics Against Enterococcus Faecalis. PloS One 9 (2), e89209. doi: 10.1371/ journal.pone.0089209

Vasconcelos, N. G., Croda, J., and Simionatto, S. (2018). Antibacterial Mechanisms of Cinnamon and its Constituents: A Review. Microb. Pathog. 120, 198-203. doi: 10.1016/j.micpath.2018.04.036

Velmourougane, K., and Prasanna, R. (2017). Influence of L-Amino Acids on Aggregation and Biofilm Formation in Azotobacter Chroococcum and Trichoderma Viride. J. Appl. Microbiol. 123 (4), 977-991. doi: 10.1111/jam.13534

Verderosa, A. D., de la Fuente-Núñez, C., Mansour, S. C., Cao, J., Lu, T. K., Hancock, R. E. W., et al. (2017). Ciprofloxacin-Nitroxide Hybrids With Potential for Biofilm Control. Eur. J. Med. Chem. 138, 590-601. doi: 10.1016/j.ejmech.2017.06.058

Verderosa, A. D., Dhouib, R., Fairfull-Smith, K. E., and Totsika, M. (2019a). Nitroxide Functionalized Antibiotics Are Promising Eradication Agents Against Staphylococcus Aureus Biofilms. Antimicrob. Agents Chemother. 64 (1), e01685-19. doi: 10.1128/AAC.01685-19

Verderosa, A. D., Harris, J., Dhouib, R., Totsika, M., and Fairfull-Smith, K. E. (2019b). Eradicating Uropathogenic Escherichia Coli Biofilms With a Ciprofloxacin-Dinitroxide Conjugate. Medchemcomm 10 (5), 699-711. doi: 10.1039/C9MD00062C

Verderosa, A. D., Mansour, S. C., de la Fuente-Núñez, C., Hancock, R. E., and Fairfull-Smith, K. E. (2016). Synthesis and Evaluation of CiprofloxacinNitroxide Conjugates as Anti-Biofilm Agents. Molecules 21 (7), 841. doi: 10.3390/molecules 21070841

Verderosa, A. D., Totsika, M., and Fairfull-Smith, K. E. (2019c). Bacterial Biofilm Eradication Agents: A Current Review. Front. Chem. 7, 824. doi: 10.3389/ fchem.2019.00824

Vermote, A., Brackman, G., Risseeuw, M. D., Vanhoutte, B., Cos, P., Van Hecke, K., et al. (2016). Hamamelitannin Analogues That Modulate Quorum Sensing as Potentiators of Antibiotics Against Staphylococcus Aureus. Angew Chem. Int. Ed Engl. 55 (22), 6551-6555. doi: 10.1002/anie.201601973

Vestby, L. K., Grønseth, T., Simm, R., and Nesse, L. L. (2020). Bacterial Biofilm and its Role in the Pathogenesis of Disease. Antibiotics (Basel) 9 (2), 59. doi: 10.3390/antibiotics9020059

Vishwakarma, V. (2020). Impact of Environmental Biofilms: Industrial Components and its Remediation. J. Basic Microbiol. 60 (3), 198-206. doi: 10.1002/jobm.201900569

Vuotto, C., and Donelli, G. (2019). Novel Treatment Strategies for Biofilm-Based Infections. Drugs 79 (15), 1635-1655. doi: 10.1007/s40265-019-01184-z

Wakabayashi, H., Yamauchi, K., Kobayashi, T., Yaeshima, T., Iwatsuki, K., and Yoshie, H. (2009). Inhibitory Effects of Lactoferrin on Growth and Biofilm Formation of Porphyromonas Gingivalis and Prevotella Intermedia. Antimicrob. Agents Chemother. 53 (8), 3308-3316. doi: 10.1128/AAC.01688-08

Wang, P. G., Xian, M., Tang, X., Wu, X., Wen, Z., Cai, T., et al. (2002). Nitric Oxide Donors: Chemical Activities and Biological Applications. Chem. Rev. 102 (4), 1091-1134. doi: 10.1021/cr0000401 
Warraich, A. A., Mohammed, A. R., Perrie, Y., Hussain, M., Gibson, H., and Rahman, A. (2020). Evaluation of Anti-Biofilm Activity of Acidic Amino Acids and Synergy With Ciprofloxacin on Staphylococcus Aureus Biofilms. Sci. Rep. 10 (1), 9021. doi: 10.1038/s41598-020-66082-x

Webert, K. E., Vanderzwan, J., Duggan, M., Scott, J. A., McCormack, D. G., Lewis, J. F., et al. (2000). Effects of Inhaled Nitric Oxide in a Rat Model of Pseudomonas Aeruginosa Pneumonia. Crit. Care Med. 28 (7), 2397-2405. doi: 10.1097/00003246-200007000-00035

Wei, W., Bing, W., Ren, J., and Qu, X. (2015). Near Infrared-Caged D-Amino Acids Multifunctional Assembly for Simultaneously Eradicating Biofilms and Bacteria. Chem. Commun. (Camb) 51 (63), 12677-12679. doi: 10.1039/ C5CC04729C

Weinberger, B., Laskin, D. L., Heck, D. E., and Laskin, J. D. (2001). The Toxicology of Inhaled Nitric Oxide. Toxicol. Sci. 59 (1), 5-16. doi: 10.1093/toxsci/59.1.5

Williams, D. E., and Boon, E. M. (2019). Towards Understanding the Molecular Basis of Nitric Oxide-Regulated Group Behaviors in Pathogenic Bacteria. J. Innate Immun. 11 (3), 205-215. doi: 10.1159/000494740

Wu, Y., Quan, X., Si, X., and Wang, X. (2016). A Small Molecule Norspermidine in Combination With Silver Ion Enhances Dispersal and Disinfection of MultiSpecies Wastewater Biofilms. Appl. Microbiol. Biotechnol. 100 (12), 5619-5629. doi: 10.1007/s00253-016-7394-y

Yang, H., Wang, M., Yu, J., and Wei, H. (2015). Aspartate Inhibits Staphylococcus Aureus Biofilm Formation. FEMS Microbiol. Letters 362 (7), fnv025. doi: 10.1093/femsle/fnv025

Yang, T., Zelikin, A. N., and Chandrawati, R. (2018). Progress and Promise of Nitric Oxide-Releasing Platforms. Adv. Sci. (Weinh) 5 (6), 1701043. doi: 10.1002/advs.201701043

Yasir, M., Dutta, D., and Willcox, M. D. P. (2020). Activity of Antimicrobial Peptides and Ciprofloxacin Against Pseudomonas Aeruginosa Biofilms. Molecules 25 (17), 3843. doi: 10.3390/molecules 25173843
Zhang, Y., Fu, Y., Yu, J., Ai, Q., Li, J., Peng, N., et al. (2015). Synergy of Ambroxol With Vancomycin in Elimination of Catheter-Related Staphylococcus Epidermidis Biofilm In Vitro and In Vivo. J. Infect. Chemother. 21 (11), 808-815. doi: 10.1016/j.jiac.2015.08.017

Zhao, Y., Liu, D., Huang, W., Yang, Y., Ji, M., Nghiem, L. D., et al. (2019). Insights Into Biofilm Carriers for Biological Wastewater Treatment Processes: Current State-of-the-Art, Challenges, and Opportunities. Bioresour Technol. 288, 121619. doi: 10.1016/j.biortech.2019.121619

Zilm, P. S., Butnejski, V., Rossi-Fedele, G., Kidd, S. P., Edwards, S., and Vasilev, K. (2017). D-Amino Acids Reduce Enterococcus Faecalis Biofilms In Vitro and in the Presence of Antimicrobials Used for Root Canal Treatment. PloS One 12 (2), e0170670. doi: 10.1371/journal.pone.0170670

Conflict of Interest: The authors declare that the research was conducted in the absence of any commercial or financial relationships that could be construed as a potential conflict of interest.

Publisher's Note: All claims expressed in this article are solely those of the authors and do not necessarily represent those of their affiliated organizations, or those of the publisher, the editors and the reviewers. Any product that may be evaluated in this article, or claim that may be made by its manufacturer, is not guaranteed or endorsed by the publisher.

Copyright (c) 2022 Hawas, Verderosa and Totsika. This is an open-access article distributed under the terms of the Creative Commons Attribution License (CC BY). The use, distribution or reproduction in other forums is permitted, provided the original author(s) and the copyright owner(s) are credited and that the original publication in this journal is cited, in accordance with accepted academic practice. No use, distribution or reproduction is permitted which does not comply with these terms. 